\title{
Gravitational wave burst signal from core collapse of rotating stars
}

\author{
Harald Dimmelmeier, ${ }^{1, *}$ Christian D. Ott, ${ }^{2,3,+}$ Andreas Marek, ${ }^{4, *}$ and H.-Thomas Janka ${ }^{4, \S}$ \\ ${ }^{1}$ Department of Physics, Aristotle University of Thessaloniki, GR-54124 Thessaloniki, Greece \\ ${ }^{2}$ Steward Observatory and Department of Astronomy, University of Arizona, Tucson, Arizona 85721, USA \\ ${ }^{3}$ Theoretical Astrophysics, Mailcode 130-33, California Institute of Technology, Pasadena, California 91125, USA \\ ${ }^{4}$ Max Planck Institute for Astrophysics, Karl-Schwarzschild-Str. 1, D-85741 Garching, Germany
}

(Received 30 June 2008; published 19 September 2008)

\begin{abstract}
We present results from detailed general relativistic simulations of stellar core collapse to a protoneutron star, using two different microphysical nonzero-temperature nuclear equations of state as well as an approximate description of deleptonization during the collapse phase. Investigating a wide variety of rotation rates and profiles as well as masses of the progenitor stars and both equations of state, we confirm in this very general setup the recent finding that a generic gravitational wave burst signal is associated with core bounce, already known as type I in the literature. The previously suggested type II (or "multiplebounce") waveform morphology does not occur. Despite this reduction to a single waveform type, we demonstrate that it is still possible to constrain the progenitor and postbounce rotation based on a combination of the maximum signal amplitude and the peak frequency of the emitted gravitational wave burst. Our models include to sufficient accuracy the currently known necessary physics for the collapse and bounce phase of core-collapse supernovae, yielding accurate and reliable gravitational wave signal templates for gravitational wave data analysis. In addition, we assess the possibility of nonaxisymmetric instabilities in rotating nascent proto-neutron stars. We find strong evidence that in an iron core-collapse event the postbounce core cannot reach sufficiently rapid rotation to become subject to a classical barmode instability. However, many of our postbounce core models exhibit sufficiently rapid and differential rotation to become subject to the recently discovered dynamical instability at low rotation rates.
\end{abstract}

DOI: 10.1103/PhysRevD.78.064056

\section{INTRODUCTION}

The final event in the life of a massive star is the catastrophic collapse of its central, electron-degenerate core composed of iron-peak nuclei. When silicon shell burning pushes the iron core over its effective Chandrasekhar mass, collapse is initiated by a combination of electron capture and photo-disintegration of heavy nuclei, both leading to a depletion of central pressure support. Massive stars in the approximate mass range of about 10 to 100 solar masses $\left(M_{\odot}\right)$ experience such a collapse phase until their homologously contracting [1,2] inner core reaches densities near and above nuclear saturation density where the nuclear equation of state (EoS) stiffens, leading to an almost instantaneous rebound of the inner core (core bounce) into the still supersonically infalling outer core. The hydrodynamic supernova shock is born, travels outward in radius and mass, but rapidly loses its kinetic energy to the dissociation of infalling iron-group nuclei and to neutrinos that deleptonize the immediate postshock material and stream off from these regions quasi-freely. The shock stalls, turns into an accretion shock and must be revived to produce the observable explosion associated with a core-collapse supernova. Mechanisms of shock

\footnotetext{
*harrydee@mpa-garching.mpg.de

${ }^{+}$cott@as.arizona.edu; cott@tapir.caltech.edu

amarek@mpa-garching.mpg.de

${ }^{\S}$ thj@mpa-garching.mpg.de
}

PACS numbers: 04.25.D-, 02.70.Bf, 04.30.Db, 97.60.Bw

revival are still under debate (a recent review is presented in [3], but see also [4-6]) and may involve heating of the postshock region by neutrinos, multidimensional hydrodynamic instabilities of the accretion shock, in the postshock region, and/or in the proto-neutron star, rotation, magnetic fields, and nuclear burning. If the shock is not revived, black-hole formation (on a timescale of $\sim 1-2 \mathrm{~s}$ [7]) is inevitable and the stellar collapse event may remain undetected by conventional astronomy or, perhaps, appear as a gamma-ray burst if the progenitor star has a compact enough envelope and sufficiently rapid rotation in its central regions $[8,9]$.

Conventional astronomy can constrain core-collapse supernova theory and the supernova explosion mechanism via secondary observables only, e.g., the explosion energy, ejecta morphology, nucleosynthesis yields, residue neutron star or black-hole mass and proper motion, and pulsar magnetic fields. Neutrinos and gravitational waves, on the other hand, are emitted deep inside the supernova core and travel to observers on Earth practically unscathed by intervening material. They can act as messengers to provide first-hand and live dynamical information on the intricate multidimensional dynamics of the proto-neutron star and postshock region and may constrain directly the core-collapse supernova mechanism. Importantly, corecollapse events that do not produce the canonical observational astronomical signature or whose observational display is shrouded from view can still be observed in 
neutrinos and gravitational waves if occurring sufficiently close to Earth.

Gravitational waves, in contrast to neutrinos, have not yet been observed directly, but an international array of gravitational wave observatories (see, e.g., [10]) is active and taking data. Since gravitational waves from astrophysical sources are expected to be weak, their detection is notoriously difficult and involves extensive signal processing and detailed analysis of the detector output. Chances for the detection of an astrophysical event of gravitational wave emission are significantly enhanced if accurate theoretical knowledge of the expected gravitational wave signature from such an event is at hand.

Theoretical predictions of the gravitational wave signature from a core-collapse supernova are complicated, since the emission mechanisms are very diverse. While the prospective gravitational wave burst signal from the collapse, bounce, and the very early postbounce phase is present only when the core rotates [11-18], gravitational wave signals with sizeable amplitudes can also be expected from convective motions at postbounce times, instabilities of the standing accretion shock, anisotropic neutrino emission, excitation of various oscillations in the protoneutron star, or nonaxisymmetric rotational instabilities [17,19-23].

In the observational search for gravitational waves from merging black hole or neutron star binaries, powerful data analysis algorithms such as matched filtering are applicable, as the waveform from the inspiral phase can be modeled with high accuracy (see, e.g., [24]) and gravitational wave data analysts already have access to robust template waveforms that depend only on a limited number of macroscopic parameters. In contrast, the complete gravitational wave signature of a core-collapse supernova cannot be predicted with template-level accuracy as the postbounce dynamics involve chaotic processes (turbulence, [magneto-] hydrodynamic instabilities) that are sensitive not only to a multitude of precollapse parameters, but also to small-scale perturbations of any of the hydrodynamic variables.

While the complete supernova gravitational wave signature may remain inaccessible to template-based data analysis, a number of individual constituent emission processes, in particular, those involving coherent global bulk dynamics and/or rotation, allow, in principle, for accurate and robust waveform predictions that may be applied to template-based searches. Rotating core collapse and core bounce as well as pulsations or nonaxisymmetric rotational deformations of a proto-neutron star constitute this group of processes. Among them, rotating collapse and bounce is the historically most extensively studied case (see, e.g., [25] for a historical review) and may be the most promising for becoming robustly predictable in its gravitational wave emission. Yet, to date, the gravitational wave signal from rotating stellar core collapse and bounce has not been predicted with the desired accuracy and robustness.
These deficiencies of previous simulations result from the fact that the physically realistic modeling of core collapse requires a general relativistic description of consistently coupled gravity and hydrodynamics in conjunction with a microphysical treatment of the sub- and supernuclear EoS, electron capture on heavy nuclei and free protons, and neutrino radiation transport. Only very few multidimensional general relativistic codes have recently begun to approach these requirements $[17,18]$. In addition, the properties of the EoS around and above nuclear density are not very well constrained by theory or experiments. The same applies to the rotation rate and angular velocity profile of the progenitor core, which are also not directly accessible by observation and very difficult to model numerically in stellar evolution codes. Furthermore, variations with progenitor structure and mass are to be expected. Therefore, the influence of rotation and progenitor structure on the collapse and bounce dynamics and thus the gravitational wave burst signal must be investigated by extensive and computationally expensive parameter studies.

Previous parameter studies have considered a large variety of rotation rates and progenitor core configurations, but generally ignored important microphysical aspects and/or the influence of general relativity. Mönchmeyer et al. [12] performed axisymmetric Newtonian calculations with progenitor models from stellar evolutionary studies. They employed the microphysical nuclear EoS of Hillebrandt and Wolff [26] and included deleptonization via a neutrino leakage scheme and electron capture on free protons. Capture on heavy nuclei was neglected, which resulted in a too high electron fraction $Y_{e}$ at core bounce and a consequently overestimated inner core mass [2,27]. In that study a limited set of four calculations was computed and two qualitatively and quantitatively different types of gravitational wave burst signals were identified. Their morphology can be classified alongside with the collapse and bounce dynamics: Type I signals are emitted when the collapse of the quasihomologously contracting inner core is not strongly influenced by rotation, but stopped by a pressure-dominated bounce due to the stiffening of the EoS near nuclear density $\rho_{\text {nuc }} \approx 2 \times 10^{14} \mathrm{~g} \mathrm{~cm}^{-3}$, where the adiabatic index $\gamma_{\text {eos }}$ rises above $4 / 3$. This leads to a bounce with a maximum core density $\rho_{\max } \geq \rho_{\text {nuc }}$. Type II signals occur when centrifugal forces, which grow during contraction owing to angular momentum conservation, are sufficiently strong to halt the collapse, resulting in consecutive (typically multiple) centrifugal bounces with intermediate coherent re-expansion of the inner core, seen as density drops by sometimes more than an order of magnitude; thus here $\rho_{\text {max }}<\rho_{\text {nuc }}$ after bounce. Type I and II dynamics and waveforms were also found in the more recent Newtonian studies by Kotake et al. [15], who employed a more complete leakage/capture scheme, but still obtained too high $Y_{e}$ at bounce, and by Ott et al. [16], who performed an extensive parameter study and for the first time also 
considered variations in progenitor star structure, but neglected deleptonization during collapse.

Zwerger and Müller [13] carried out an extensive twodimensional Newtonian study of rotating collapse of idealized polytropes in rotational equilibrium [28] with a simplified hybrid EoS, consisting of a polytropic and a thermal component [29]. Electron capture during collapse was mimicked by an instantaneous lowering of the adiabatic index $\gamma_{\text {eos }}$ from its initial value of $4 / 3$ to trigger the onset of collapse. At $\rho_{\text {nuc }}$, the adiabatic index was raised to $\gtrsim 2$ to qualitatively model the stiffening of the nuclear EoS. Zwerger and Müller also obtained the previously suggested signal types and introduced type III signals that appear in a pressure-dominated bounce when the inner core has a very small mass due to very efficient electron capture (approximated in [13] via a $\gamma_{\text {eos }} \leqslant 1.29$ in their hybrid EoS). Obergaulinger et al. [30] also employed the hybrid EoS, but included magnetic fields. They introduced the additional dynamics/signal type $I V$, which occurs only in the case of very strong precollapse core magnetization. They found that weak to moderate core magnetization in agreement with predictions from stellar evolution theory (see, e.g., [31]) has little effect on the collapse and bounce dynamics and the resulting gravitational wave signal. This finding is in agreement with [32] (see also [5,33]), where magnetorotational collapse simulations were performed, a smaller model set was considered, but the neutrino leakage scheme of [15] was employed, and it made use of two different microphysical EoSs to study the EoS dependence of the collapse dynamics and gravitational wave signal.

The first extensive set of general relativistic simulations of rotating iron core collapse to a proto-neutron star were presented by Dimmelmeier et al. [14], who employed an analytic hybrid EoS and polytropic precollapse models in rotational equilibrium as initial data (but see also the pioneering early work of [34]). These simulations were subsequently confirmed in [25,35-37]. Dimmelmeier et al. studied a subset of the models in [13] in the same parameter space of rotation rate and degree of differential rotation, and found that general relativistic effects counteract centrifugal support and shift the occurrence of type II dynamics and wave signals to a higher precollapse rotation rate at a fixed degree of differential rotation.

Recently, new general relativistic simulations of rotating core collapse in two and three dimensions were carried out by Ott et al. $[17,25,38]$ who included the microphysical EoS of Shen et al. [39], precollapse models from stellar evolutionary calculations as well as an approximate deleptonization scheme [40]. The results of these calculations indicate that the gravitational wave burst signal associated with rotating core collapse is exclusively of type I. In addition, the simulations showed that rotating stellar iron cores stay axisymmetric throughout collapse and bounce, and only at postbounce times develop nonaxisymmetric features.
In a general relativistic two-dimensional follow-up study, Dimmelmeier et al. [18,41] considerably extended the number of models and comprehensively explored a wide parameter space of precollapse rotational configurations. Even for this more general setup they found gravitational wave signals solely of type I form, although for rapid precollapse rotation some of their models experience a core bounce due to centrifugal forces only, which however is always a single centrifugal bounce rather than the multiple ones observed in earlier work (see, e.g., $[13,14,16])$. They identified the physical conditions that lead to the emergence of this generic gravitational wave signal type and quantified their relative influence. These results strongly suggest that the waveform of the gravitational wave burst signal from the collapse of rotating iron cores in a core-collapse event is much more generic than previously anticipated.

In this work, we extend the above study of the gravitational wave signal from rotating core collapse and consider not only variations in the precollapse rotational configuration, but also in progenitor structure and nuclear EoS. In this way, we carry out the to-date largest and most complete parameter study of rotating stellar core collapse that includes all the (known) necessary physics to produce reliable predictions of the gravitational wave signal associated with rotating collapse and bounce. All our computed gravitational wave signals are made available to the detector data analysis community in a freely accessible waveform catalog [42].

We perform a large number of two-dimensional simulations with our general relativistic core-collapse code COCONUT and employ 11.2, 15.0, 20.0, and 40.0M $\odot$ (masses at zero-age main sequence) precollapse stellar models from the stellar evolutionary studies of Heger et al. [31,43]. In addition to the EoS by Shen et al. [39] used in our previous studies, we also calculate models with the EoS by Lattimer and Swesty [44]. We describe in detail and explain comprehensively the qualitative and quantitative aspects of the collapse and bounce dynamics and the resultant gravitational wave signal. We lay out the individual effects of general relativity, deleptonization, precollapse stellar structure and rotational configuration, and nuclear EoS on the gravitational wave signature from rotating core collapse. We study the prospects for nonaxisymmetric rotational instabilities in our postbounce cores, which could lead to an enhancement of the gravitational wave signature. Furthermore, we set our model gravitational radiation waveforms in context with present and future detector technology and assess their detectability.

This paper is organized as follows: In Sec. II, we introduce our treatment of the general relativistic spacetime curvature and hydrodynamics equations. Furthermore, we introduce our variants of the two microphysical EoS we employ, the scheme for deleptonization and neutrino 
pressure contributions, our precollapse model set, and the gravitational wave extraction technique employed. Section III discusses the numerical methods used in the COCONUT code and the computational grid setup for the simulations presented in this paper. In Sec. IV, we present the collapse dynamics and waveform morphology of our simulated models, while in Sec. V, we investigate the stratification of the postbounce core and its impact on the gravitational wave signal. The detection prospects for the gravitational wave burst from core bounce are discussed in Sec. VI, while the rotational configuration of the protoneutron star and its susceptibility to nonaxisymmetric rotational instabilities are examined in Sec. VII. Finally, in Sec. VIII, we summarize and discuss our results.

Throughout the paper we use a spacelike signature $(-,+,+,+)$ and units in which $c=G=1$. Greek indices run from 0 to 3 , Latin indices from 1 to 3 , and we adopt the standard Einstein summation convention.

\section{PHYSICAL MODEL AND EQUATIONS}

\section{A. General relativistic hydrodynamics}

We adopt the Arnowitt-Deser-Misner (ADM) $3+1$ formalism of general relativity to foliate the spacetime endowed with a four metric $g_{\mu \nu}$ into spacelike hypersurfaces [45]. In this approach the line element reads

$$
d s^{2}=-\alpha^{2} d t^{2}+\gamma_{i j}\left(d x^{i}+\beta^{i} d t\right)\left(d x^{j}+\beta^{j} d t\right),
$$

where $\alpha$ is the lapse function, $\beta^{i}$ is the shift vector, and $\gamma_{i j}$ is the spatial three metric induced in each hypersurface.

The hydrodynamic evolution of a perfect fluid in general relativity with four velocity $u^{\mu}$, rest-mass current $J^{\mu}=$ $\rho u^{\mu}$, and stress-energy tensor $T^{\mu \nu}=\rho h u^{\mu} u^{\nu}+P g^{\mu \nu}$ is determined by a system of local conservation equations

$$
\nabla_{\mu} J^{\mu}=0, \quad \nabla_{\mu} T^{\mu \nu}=0,
$$

where $\nabla_{\mu}$ denotes the covariant derivative with respect to the four metric. Here, $\rho$ is the rest-mass density, $h=1+$ $\epsilon+P / \rho$ is the specific enthalpy, $P$ is the fluid pressure, and the three velocity with respect to an Eulerian observer moving orthogonally to the spacelike hypersurfaces is given by $v^{i}=u^{i} /\left(\alpha u^{0}\right)+\beta^{i} / \alpha$. We define a set of conserved variables as

$$
D=\rho W, \quad S^{i}=\rho h W^{2} v^{i}, \quad \tau=\rho h W^{2}-P-D .
$$

In the above expressions, $W=\alpha u^{0}$ is the Lorentz factor, which satisfies the relation $W=1 / \sqrt{1-v_{i} v^{i}}$.

The local conservation laws (2) are written as a firstorder, flux-conservative system of hyperbolic equations [46]

$$
\frac{\partial \sqrt{\gamma} \boldsymbol{U}}{\partial t}+\frac{\partial \sqrt{-g} \boldsymbol{F}^{i}}{\partial x^{i}}=\sqrt{-g} \boldsymbol{S},
$$

with

$$
\begin{gathered}
\boldsymbol{U}=\left[D, S_{j}, \tau, D Y_{e}\right], \\
\boldsymbol{F}^{i}=\left[D \hat{\boldsymbol{v}}^{i}, S_{j} \hat{\boldsymbol{v}}^{i}+\delta_{j}^{i} P, \tau \hat{\boldsymbol{v}}^{i}+P \boldsymbol{v}^{i}, D Y_{e} \hat{\boldsymbol{v}}^{i}\right] \\
\boldsymbol{S}=\left[0, \frac{T^{\mu \nu}}{2} \frac{\partial g_{\mu \nu}}{\partial x^{j}}-\frac{\partial P_{\nu}}{\partial x^{j}}, T^{00}\left(K_{i j} \beta^{i} \beta^{j}-\beta^{i} \frac{\partial \alpha}{\partial x^{i}}\right)\right. \\
\left.+T^{0 i}\left(2 K_{i j} \beta^{j}-\frac{\partial \alpha}{\partial x^{i}}\right)+T^{i j} K_{i j}-v^{i} \frac{\partial P_{\nu}}{\partial x^{i}}, 0\right] .
\end{gathered}
$$

Here, $\hat{v}^{i}=v^{i}-\beta^{i} / \alpha$, and $g$ and $\gamma$ are the determinant of $g_{\mu \nu}$ and $\gamma_{i j}$, respectively, with $\sqrt{-g}=\alpha \sqrt{\gamma} . \Gamma_{\mu \nu}^{\lambda}$ are the four-Christoffel symbols. Since we use a microphysical EoS that requires information on the local electron fraction per baryon $Y_{e}$, we add an advection equation for the quantity $D Y_{e}$ to the standard form of the conservation Eq. (4). The radiation stress due to the neutrino pressure $P_{\nu}$ (as defined in Sec. II D), is included in the form of an additive term in the source of both the momentum and energy equations. Note also that here we use an analytically equivalent reformulation of the energy source term in contrast to the one presented in [14].

\section{B. Metric equations in the conformal flatness approximation}

Using the ADM $3+1$ formalism, the Einstein equations split into a coupled set of first-order evolution equations for the three-metric $\gamma_{i j}$ and the extrinsic curvature $K_{i j}$

$$
\begin{gathered}
\partial_{t} \gamma_{i j}=-2 \alpha K_{i j}+\nabla_{i} \beta_{j}+\nabla_{j} \beta_{i}, \\
\partial_{t} K_{i j}=-\nabla_{i} \nabla_{j} \alpha+\alpha\left(R_{i j}-2 K_{i k} K_{j}^{k}\right)+\beta^{k} \nabla_{k} K_{i j} \\
+K_{i k} \nabla_{j} \beta^{k}+K_{j k} \nabla_{i} \beta^{k} \\
-8 \pi \alpha\left(S_{i j}-\frac{\gamma_{i j}}{2}\left(S_{k}^{k}-\rho_{\mathrm{ADM}}\right)\right)
\end{gathered}
$$

and constraint equations,

$$
\begin{gathered}
0=R-K_{i j} K^{i j}-16 \pi \rho_{\mathrm{ADM}}, \\
0=\nabla_{i} K^{i j}-8 \pi S^{j} .
\end{gathered}
$$

In the above equations, $\nabla_{i}$ is the covariant derivative with respect to the three metric $\gamma_{i j}, R_{i j}$ is the three-Ricci tensor, and $R$ is the scalar three curvature. The projection of the stress-energy tensor onto the spatial hypersurface is $S_{i j}=$ $\rho h W^{2} v_{i} v_{j}+\gamma_{i j} P$, the ADM energy density is given by $\rho_{\mathrm{ADM}}=\rho h W^{2}-P$, and $S^{j}=\rho h W^{2} v^{i}$ is the momentum density. In addition, we have chosen the maximal slicing condition for which the trace of the extrinsic curvature vanishes: $K=0$.

In order to simplify the ADM metric equations and to ameliorate the stability properties when numerically solving those equations, we employ the conformal flatness 
condition (CFC) introduced in [47] and first used in a pseudo-evolutionary context in [48]. In this approximation the spatial three metric is replaced by the conformally flat three metric $\gamma_{i j}=\phi^{4} \hat{\gamma}_{i j}$, where $\hat{\gamma}_{i j}$ is the flat-space metric and $\phi$ is the conformal factor. Then the metric Eqs. (8)(11) reduce to a set of elliptic equations for $\phi, \alpha$, and $\beta^{i}$,

$$
\begin{gathered}
\hat{\Delta} \phi=-2 \pi \phi^{5}\left(E+\frac{K_{i j} K^{i j}}{16 \pi}\right), \\
\hat{\Delta}(\alpha \phi)=2 \pi \alpha \phi^{5}\left(E+2 S+\frac{7 K_{i j} K^{i j}}{16 \pi}\right), \\
\hat{\Delta} \beta^{i}=16 \pi \alpha \phi^{4} S^{i}+2 \phi^{10} K^{i j} \hat{\nabla}_{j} \frac{\alpha}{\phi^{6}}-\frac{1}{3} \hat{\nabla}^{i} \hat{\nabla}_{k} \beta^{k},
\end{gathered}
$$

where $\hat{\Delta}$ and $\hat{\nabla}$ are the Laplace and covariant derivative operators associated with the flat three metric, and $S=$ $\gamma^{i j} S_{i j}$. The CFC metric Eqs. (12)-(14) do not contain explicit time derivatives, and thus the metric components are evaluated in a fully constrained approach.

Imposing CFC in a spherically symmetric spacetime is equivalent to solving the exact Einstein equations. For nonspherical configurations the CFC approximation may be roughly regarded as full general relativity without the dynamical degrees of freedom of the gravitational field that correspond to the gravitational wave content [49]. However, even spacetimes that do not contain gravitational waves can be not conformally flat. A prime example are the spacetime of a Kerr black hole [50] or rotating fluids in equilibrium. For rapidly rotating models of stationary neutron stars the deviation of certain metric components from conformal flatness has been shown to reach up to $\sim 5 \%$ in extreme cases [51], while the oscillation frequencies of such models typically deviate even less from the corresponding values obtained in full general relativistic simulations [52]. In the context of rotating stellar core collapse the excellent quality of the CFC approximation has been demonstrated extensively $[17,35,36]$.

Because of its fully constrained nature, the CFC approximation permits a straightforward and numerically more robust implementation of the metric equations in coordinate systems containing coordinate singularities (e.g., spherical polar coordinates) compared with a Cauchy free-evolution scheme. Furthermore, by definition it allows no constraint violations, which is a significant benefit in cases where a perturbation is added to the initial data. More details on the CFC equations can be found in, e.g., [14].

\section{Equations of state}

In our simulations we employ two tabulated nonzerotemperature equations of state, the one by Shen et al. [39,53] (Shen EoS), and the one by Lattimer and Swesty [44] (LS EoS). The LS EoS is based on a compressible liquid-drop model [54]. The transition from inhomogeneous to homogeneous matter is established by a Maxwell construction, and the nucleon-nucleon interactions are expressed by a Skyrme force. In our version of this EoS, the incompressibility modulus of bulk nuclear matter is taken to be $180 \mathrm{MeV}$ and the symmetry energy parameter has a value of $29.3 \mathrm{MeV}$. In contrast, the Shen EoS is based on a relativistic mean field model and is extended with the Thomas-Fermi approximation to describe the homogeneous phase of matter as well as the inhomogeneous matter composition. The parameter for the incompressibility of nuclear matter is $281 \mathrm{MeV}$ and the symmetry energy has a value of $36.9 \mathrm{MeV}$.

Both EoSs employed in this study are the same as in Marek et al. [55] and include contributions of baryons, electrons, positrons, and photons. Furthermore, in this study the LS EoS has been extended to densities below $\rho=5.8 \times 10^{7} \mathrm{~g} \mathrm{~cm}^{-3}$ by a smooth transition to the Shen EoS, which is tabulated down to $\rho=6.4 \times 10^{5} \mathrm{~g} \mathrm{~cm}^{-3}$.

The microphysical EoS returns the fluid pressure (and additional thermodynamic quantities) as a function of ( $\rho, T, Y_{e}$ ), where $T$ is the temperature. Since the hydrodynamic Eq. (4) operate on the specific internal energy $\epsilon$, we determine the corresponding temperature $T$ iteratively with a Newton-Raphson scheme and the EoS table. All interpolations are carried out in trilinear fashion and the tables are sufficiently densely spaced to lead to an artificial entropy increase in an adiabatic collapse by not more than $\sim 2 \%$.

\section{Deleptonization and neutrino pressure}

Electron capture on free protons and heavy nuclei during collapse reduces $Y_{e}$ (i.e., "deleptonizes" the collapsing core) and consequently decreases the size of the homologously collapsing inner core that depends on the average value of $Y_{e}$ in a roughly quadratic way (see, e.g., [56]). The material of the inner core is in sonic contact and determines the dynamics and the gravitational wave signal at core bounce and in the early postbounce phases. Hence, deleptonization has a direct influence on the collapse dynamics and the gravitational wave signal, and thus it is essential to include deleptonization during collapse.

Since multidimensional radiation-hydrodynamics calculations in general relativity are not yet computationally feasible, in our simulations we make use of a recently proposed approximative scheme [40], where deleptonization is parametrized based on data from detailed spherically symmetric calculations with Boltzmann neutrino transport, for which (as in [18]) we take the latest available electron capture rates [57]. Following the main assumption in [40] that the local electron fraction for each fluid element during the contraction phase can be modeled rather accurately by a dependence on the density only, these simulations yield a universal relation $\bar{Y}_{e}(\rho)$. Furthermore, we find that this relation varies only slightly with progenitor mass, as shown in Fig. 1, where models with identical progenitor but different EoS have the same color, but 


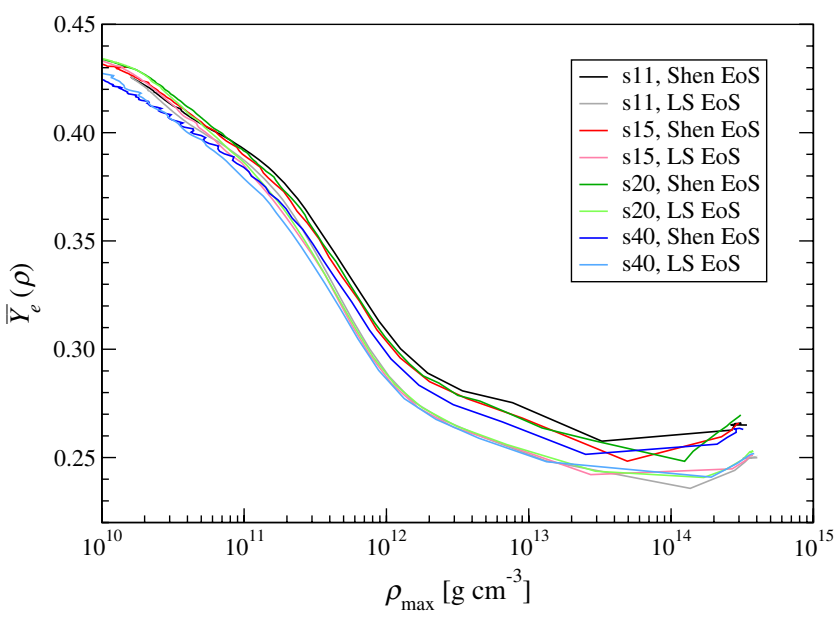

FIG. 1 (color online). Electron fraction $\bar{Y}_{e}$ obtained from detailed spherically symmetric calculations with Boltzmann neutrino transport versus the maximum density $\rho_{\max }$ in the collapsing core. The EoS is encoded in dark hues for the Shen EoS and light hues for the LS EoS with the basis color specifying the progenitor mass.

different hues (e.g., dark green versus light green for the s20 progenitor). Consequently, we utilize the $20.0 M_{\odot}$ progenitor to create such a profile $\bar{Y}_{e}(\rho)$ for each of the two EoSs. This profile is then used to correct the value of $Y_{e}$ obtained from the advection by an amount

$$
\Delta Y_{e}=\min \left[0, \bar{Y}_{e}(\rho)-Y_{e}\right]
$$

after each time integration step. This procedure assures that $Y_{e}$ approaches the phenomenological input profile $\bar{Y}_{e}(\rho)$ with the constraint that $\Delta Y_{e}$ must be negative. Accordingly, in order to model the entropy loss by neutrinos escaping the collapsing core, for densities below an adopted neutrino trapping density $\rho_{\text {tr }}=2 \times 10^{12} \mathrm{~g} \mathrm{~cm}^{-3}$ the internal specific energy $\epsilon$ is re-adjusted at constant $\rho$ and $Y_{e}$ such that the specific entropy per baryon $s$ is changed by

$$
\Delta s=-\Delta Y_{e} \frac{\mu_{p}-\mu_{n}+\mu_{e}-E_{\nu}}{k_{\mathrm{B}} T},
$$

where $E_{\nu}=10 \mathrm{MeV}$ is an average escape energy for the neutrinos, $k_{\mathrm{B}}$ is the Boltzmann constant and where $\mu_{p}, \mu_{n}$, and $\mu_{e}$ are the proton, neutron, and electron chemical potentials, respectively. Note that when equilibrium between neutrinos and matter (i.e., $\beta$ equilibrium) is established, this balance requires $\mu_{\nu}=\mu_{p}-\mu_{n}+\mu_{e}$ for the neutrino chemical potential $\mu_{\nu}$.

We stop deleptonization at the time of core bounce (i.e., as soon as the specific entropy $s$ per baryon exceeds $3 k_{\mathrm{B}}$ at the outer boundary of the inner core). After core bounce, for lack of a simple yet accurate approximation scheme for treating the further deleptonization in the nascent protoneutron star, we advect $Y_{e}$ only passively according to the conservation Eq. (4), although this effectively prevents the factual cooling and contraction of the proto-neutron star.
In all collapse phases, however, as in [40] we approximate the pressure contribution of the neutrinos by that of an ideal Fermi gas

$$
P_{\nu}=\frac{4 \pi\left(k_{\mathrm{B}} T\right)^{4}}{3(h c)^{3}} F_{3}\left(\frac{\mu_{\nu}}{k_{\mathrm{B}} T}\right),
$$

with $F_{3}$ being the Fermi-Dirac function of order 3. The neutrino pressure is included only in the regime that is optically thick to neutrinos, which we define for densities above $\rho_{\text {tr }}$.

\section{E. Initial models}

All presupernova stellar models available to date are end products of Newtonian spherically symmetric stellar evolutionary calculations from hydrogen burning on the main sequence to the onset of core collapse by photo dissociation of heavy nuclei and electron captures (see, e.g., [58]). Here, we employ various nonrotating models of [58] with zero-age main sequence masses $M_{\text {prog }}=11.2 M_{\odot}$ (coremodel s11.2, here for simplicity labeled s11), $15.0 M_{\odot}$ (core-model s15), $20.0 M_{\odot}$ (core-model s20), and $40.0 M_{\odot}$ (core-model s40). Recently, the first presupernova models that include rotation in a one-dimensional approximate fashion have become available [31,43], and of these we select ones with $M_{\text {prog }}=15.0 M_{\odot}$ (models e15a and e15b) as well as $20.0 M_{\odot}$ (core-models e20a and e20b). All progenitors have solar metallicity (at zero-age main sequence), and we generate our initial models by taking the data obtained from stellar evolution out to a radius $R_{\mathrm{i}}$, where the density drops to a value that equals $10^{-4}$ of the initial precollapse central density $\rho_{\mathrm{c}, \mathrm{i}}$. Selected quantities that describe the properties of these stellar cores are summarized in Table I.

We set those cores that are initially nonrotating (coremodels s11, s15, s20, and s40) artificially into rotation according to the rotation law specified in [28], where the specific angular momentum $j$ is given by

$$
j=A^{2}\left(\Omega_{\mathrm{c}, \mathrm{i}}-\Omega\right) .
$$

Here, the length $A$ parametrizes the degree of differential rotation (stronger differentiality with decreasing $A$ ) and $\Omega_{\mathrm{c}, \mathrm{i}}$ is the precollapse value of the angular velocity $\Omega$ at the center. In the Newtonian limit, this reduces to

$$
\Omega=\Omega_{\mathrm{c}, \mathrm{i}} \frac{A^{2}}{A^{2}+r^{2} \sin ^{2} \theta},
$$

with $r \sin \theta$ being the distance to the rotation axis.

In order to determine the influence of different angular momentum distributions on the collapse dynamics, we parameterize the precollapse rotation of our models in terms of $A$ (A1: $A=50,000 \mathrm{~km}$, almost uniform; A2: $A=$ $1,000 \mathrm{~km}$, moderately differential; A3: $A=500 \mathrm{~km}$, strongly differential); and $\Omega_{\mathrm{c}, \mathrm{i}}$. The model nomenclature for the precollapse rotation parameters is shown in Table II. We have selected the rotational configuration of the models 
TABLE I. Properties of the iron core models used as initial data. $M_{\text {prog }}$ is the total zero-age main sequence mass of the progenitor star, $M_{\text {core }}$ and $R_{\text {core }}$ are the mass and radius of the iron core, $M_{\mathrm{i}}$ and $R_{\mathrm{i}}$ are the mass and radius of the initial model on the computational grid, and $\rho_{\mathrm{c}, \mathrm{i}}$ is the precollapse density at the center. The size of the iron core is determined by the condition that $Y_{e}$ exceeds 0.497 , while the initial model extends beyond the iron core to where the density drops to $10^{-4} \rho_{\mathrm{c}, \mathrm{i}} \cdot \rho_{\mathrm{c}, \mathrm{i}}$ deviates slightly from the original value of the models in [58] because of regridding to the more densely spaced central grid of the evolution code.

\begin{tabular}{|c|c|c|c|c|c|c|}
\hline Core model & $M_{\text {prog }}\left[M_{\odot}\right]$ & $M_{\text {core }}\left[M_{\odot}\right]$ & $R_{\text {core }}\left[10^{3} \mathrm{~km}\right]$ & $M_{\mathrm{i}}\left[M_{\odot}\right]$ & $R_{\mathrm{i}}\left[10^{3} \mathrm{~km}\right]$ & $\rho_{\mathrm{c}, \mathrm{i}}\left[10^{9} \mathrm{~g} \mathrm{~cm}^{-3}\right]$ \\
\hline s11 & 11.2 & 1.24 & 0.99 & 1.36 & 1.58 & 17.71 \\
\hline s15 & 15.0 & 1.55 & 1.94 & 1.81 & 3.88 & 6.50 \\
\hline s20 & 20.0 & 1.46 & 1.69 & 1.59 & 3.48 & 8.77 \\
\hline $\mathrm{s} 40$ & 40.0 & 1.55 & 1.62 & 2.03 & 4.60 & 3.88 \\
\hline e15a & 15.0 & 1.47 & 1.55 & 1.83 & 4.45 & 5.78 \\
\hline $\mathrm{e} 15 \mathrm{~b}$ & 15.0 & 1.40 & 1.66 & 1.56 & 3.17 & 8.04 \\
\hline e20a & 20.0 & 1.75 & 2.41 & 2.26 & 5.42 & 4.27 \\
\hline $\mathrm{e} 20 \mathrm{~b}$ & 20.0 & 1.38 & 1.35 & 1.60 & 3.18 & 7.22 \\
\hline
\end{tabular}

TABLE II. Precollapse rotation properties of the core-collapse models. $A$ is the differential rotation length scale, $\Omega_{\mathrm{c}, \mathrm{i}}$ is the precollapse angular velocity at the center, and $\beta_{\mathrm{i}}$ is the precollapse rotation rate. Note that the models e15a, e15b, e20a, and e20b have a rotation profile from the corresponding stellar evolution calculations, while onto all other models an artificial rotation profile is imposed.

\begin{tabular}{|c|c|c|c|c|c|c|c|c|c|c|c|}
\hline $\begin{array}{l}\text { Rotating core } \\
\text { model }\end{array}$ & $\begin{array}{c}A \\
{\left[10^{8} \mathrm{~cm}\right]}\end{array}$ & $\begin{array}{c}\Omega_{\mathrm{c}, \mathrm{i}} \\
{\left[\mathrm{rad} \mathrm{s}^{-1}\right]}\end{array}$ & $\begin{array}{c}\beta_{\mathrm{i}} \\
{[\%]}\end{array}$ & $\begin{array}{l}\text { Rotating core } \\
\text { model }\end{array}$ & $\begin{array}{c}A \\
{\left[10^{8} \mathrm{~cm}\right]}\end{array}$ & $\begin{array}{c}\Omega_{\mathrm{c}, \mathrm{i}} \\
{\left[\mathrm{rad} \mathrm{s}^{-1}\right]}\end{array}$ & $\begin{array}{c}\beta_{\mathrm{i}} \\
{[\%]}\end{array}$ & $\begin{array}{c}\text { Rotating core } \\
\text { model }\end{array}$ & $\begin{array}{c}A \\
{\left[10^{8} \mathrm{~cm}\right]}\end{array}$ & $\begin{array}{c}\Omega_{\mathrm{c}, \mathrm{i}} \\
{\left[\mathrm{rad} \mathrm{s}^{-1}\right]}\end{array}$ & $\begin{array}{c}\beta_{\mathrm{i}} \\
{[\%]}\end{array}$ \\
\hline s11A1O01 & 50.0 & 0.45 & 0.01 & s15A1O01 & 50.0 & 0.45 & 0.09 & e15a & - & 4.18 & 0.46 \\
\hline s11A1O05 & 50.0 & 1.01 & 0.06 & s15A1O05 & 50.0 & 1.01 & 0.45 & $\mathrm{e} 15 \mathrm{~b}$ & - & 9.93 & 2.75 \\
\hline s11A1O07 & 50.0 & 1.43 & 0.12 & s15A1O07 & 50.0 & 1.43 & 0.91 & e20a & - & 3.13 & 0.28 \\
\hline s11A1O09 & 50.0 & 1.91 & 0.22 & s15A1O09 & 50.0 & 1.91 & 1.63 & $\mathrm{e} 20 \mathrm{~b}$ & - & 11.01 & 2.16 \\
\hline s11A1O13 & 50.0 & 2.71 & 0.43 & s15A1O13 & 50.0 & 2.71 & 3.26 & & & & \\
\hline s11A2O05 & 1.0 & 2.40 & 0.16 & s15A2O05 & 1.0 & 2.40 & 0.30 & & & & \\
\hline s11A2O07 & 1.0 & 3.40 & 0.31 & s15A2O07 & 1.0 & 3.40 & 0.60 & & & & \\
\hline s11A2O09 & 1.0 & 4.56 & 0.56 & s15A2O09 & 1.0 & 4.56 & 1.09 & & & & \\
\hline s11A2O13 & 1.0 & 6.45 & 1.13 & s15A2O13 & 1.0 & 6.45 & 2.18 & & & & \\
\hline s11A2O15 & 1.0 & 7.60 & 1.57 & s15A2O15 & 1.0 & 7.60 & 3.03 & & & & \\
\hline s11A3O05 & 0.5 & 4.21 & 0.20 & s15A3O05 & 0.5 & 4.21 & 0.27 & & & & \\
\hline s11A3O07 & 0.5 & 5.95 & 0.40 & s15A3O07 & 0.5 & 5.95 & 0.53 & & & & \\
\hline s11A3009 & 0.5 & 8.99 & 0.72 & s15A3O09 & 0.5 & 8.99 & 0.96 & & & & \\
\hline s11A3O12 & 0.5 & 10.65 & 1.28 & s15A3O12 & 0.5 & 10.65 & 1.71 & & & & \\
\hline s11A3O13 & 0.5 & 11.30 & 1.44 & s15A3O13 & 0.5 & 11.30 & 1.92 & & & & \\
\hline s11A3O15 & 0.5 & 13.31 & 2.00 & s15A3O15 & 0.5 & 13.31 & 2.67 & & & & \\
\hline s20A1O01 & 50.0 & 0.45 & 0.05 & s40A1O01 & 50.0 & 0.45 & 0.13 & & & & \\
\hline s20A1O05 & 50.0 & 1.01 & 0.25 & s40A1O05 & 50.0 & 1.01 & 0.64 & & & & \\
\hline s20A1O07 & 50.0 & 1.43 & 0.50 & s40A1O07 & 50.0 & 1.43 & 1.28 & & & & \\
\hline s20A1O09 & 50.0 & 1.91 & 0.90 & s40A1O09 & 50.0 & 1.91 & 2.31 & & & & \\
\hline s20A1O13 & 50.0 & 2.71 & 1.80 & s40A1O13 & 50.0 & 2.71 & 4.62 & & & & \\
\hline s20A2O05 & 1.0 & 2.40 & 0.25 & s40A2O05 & 1.0 & 2.40 & 0.36 & & & & \\
\hline s20A2O07 & 1.0 & 3.40 & 0.50 & s40A2O07 & 1.0 & 3.40 & 0.72 & & & & \\
\hline s20A2O09 & 1.0 & 4.56 & 0.90 & s40A2O09 & 1.0 & 4.56 & 1.30 & & & & \\
\hline s20A2O13 & 1.0 & 6.45 & 1.80 & s40A2O13 & 1.0 & 6.45 & 2.60 & & & & \\
\hline s20A2O15 & 1.0 & 7.60 & 2.50 & s40A2O15 & 1.0 & 7.60 & 3.62 & & & & \\
\hline s20A3O05 & 0.5 & 4.21 & 0.25 & s40A3O05 & 0.5 & 4.21 & 0.29 & & & & \\
\hline s20A3O07 & 0.5 & 5.95 & 0.50 & s40A3O07 & 0.5 & 5.95 & 0.57 & & & & \\
\hline s20A3009 & 0.5 & 8.99 & 0.90 & s40A3O09 & 0.5 & 8.99 & 1.03 & & & & \\
\hline s20A3O12 & 0.5 & 10.65 & 1.60 & s40A3O12 & 0.5 & 10.65 & 1.84 & & & & \\
\hline s20A3O13 & 0.5 & 11.30 & 1.80 & s40A3O13 & 0.5 & 11.30 & 2.07 & & & & \\
\hline s20A3O15 & 0.5 & 13.31 & 2.50 & s40A3O15 & 0.5 & 13.31 & 2.87 & & & & \\
\hline
\end{tabular}


in such a way that for the s20 progenitor they are a representative subset of the models investigated in $[18,41]$. They reflect different properties of the collapse dynamics and the gravitational radiation waveform discussed in that work, namely, pressure-dominated bounce with or without significant postbounce convective overturn as well as single centrifugal bounce.

Note that models with the same rotation specification (but different progenitor mass or EoS) have an identical angular velocity profile, while the precollapse rotation rate $\beta_{\mathrm{i}}=T_{\mathrm{i}} /|W|_{\mathrm{i}}$, which is the precollapse ratio of rotational energy to gravitational energy, varies. We have decided to compare models with identical initial angular velocity $\Omega_{\mathrm{c}, \mathrm{i}}$ and not precollapse rotation rate $\beta_{\mathrm{i}}$, as the latter quantity is rather sensitive to the chosen core radius $R_{\text {core }}$ in the case of (almost) uniform rotation.

The models that are based on progenitor calculations including rotation (core-models e15a, e15b, e20a, and e20b) are mapped onto our computational grids under the assumption of constant rotation on cylindrical shells of constant distance to the rotation axis. We also point out that due to the one-dimensional nature, none of the considered models are in rotational equilibrium. Still, in slowly rotating initial models this effect is small and thus negligible. For more rapidly rotating models, which exhibits the strongest deviation from rotational equilibrium, the collapse proceeds more slowly due to stabilizing centrifugal forces, and hence the star has more time for the adjustment to the appropriate angular stratifications for its rate of rotation.

In this study, we focus on the collapse of massive presupernova iron cores with at most moderate differential rotation and precollapse rotation rates that except for the most slowly rotating models lead to proto-neutron stars that are probably spinning too fast to yield spin periods of cold neutron stars in agreement with observationally inferred injection periods of young pulsars into the $P / \dot{P}$ diagram [31,59]. However, they may be highly relevant in the collapsar-type gamma-ray burst scenario $[9,59,60]$.

\section{F. Gravitational wave extraction}

We employ the Newtonian quadrupole formula in the first moment of momentum density formulation as discussed, e.g., in $[14,61,62]$ to extract the gravitational waves generated by nonspherical accelerated fluid motions. It yields the quadrupole wave amplitude $A_{20}^{\mathrm{E} 2}$ as the lowest order term in a multipole expansion of the radiation field into pure-spin tensor harmonics [63]. The wave amplitude is related to the dimensionless gravitational wave strain $h$ in the equatorial plane by

$$
h=\frac{1}{8} \sqrt{\frac{15}{\pi}} \frac{A_{20}^{\mathrm{E} 2}}{r}=8.8524 \times 10^{-21} \frac{A_{20}^{\mathrm{E} 2}}{10^{3} \mathrm{~cm}} \frac{10 \mathrm{kpc}}{r},
$$

where $r$ is the distance to the emitting source.
We point out that although the quadrupole formula is not gauge invariant and is only valid in the Newtonian slowmotion limit, for gravitational waves emitted by pulsations of rotating NSs (i.e., in astrophysical situations comparable to collapsing stellar cores at bounce in terms of compactness and rotation rates) it yields results that agree very well in phase and to $\sim 10-20 \%$ in amplitude with more sophisticated methods $[61,64]$.

In order to assess the prospects for detection by current and planned interferometer detectors and to specifically address the issue of detection range and expected event rates, we calculate the dimensionless characteristic gravitational wave strain $h_{\mathrm{c}}$ of the signal according to [65]. We first perform a Fourier transform of the gravitational wave strain $h$,

$$
\hat{h}=\int_{-\infty}^{\infty} e^{2 \pi i f t} h d t .
$$

To obtain the (detector dependent) integrated characteristic signal frequency

$$
f_{\mathrm{c}}=\left(\int_{0}^{\infty} \frac{\left\langle\hat{h}^{2}\right\rangle}{S_{h}} f d f\right)\left(\int_{0}^{\infty} \frac{\left\langle\hat{h}^{2}\right\rangle}{S_{h}} d f\right)^{-1}
$$

and the integrated characteristic signal strain

$$
h_{\mathrm{c}}=\left(3 \int_{0}^{\infty} \frac{S_{h \mathrm{c}}}{S_{h}}\left\langle\hat{h}^{2}\right\rangle f d f\right)^{1 / 2},
$$

the power spectral density $S_{h}$ of the detector is needed (with $S_{h \mathrm{c}}=S_{h}\left(f_{\mathrm{c}}\right)$ ). We approximate the average $\left\langle\hat{h}^{2}\right\rangle$ over randomly distributed angles by $\hat{h}^{2}$, assuming optimal orientation of the interferometer detector. From Eqs. (22) and (23) the signal-to-noise ratio can be computed as SNR = $h_{\mathrm{c}} /\left[h_{\mathrm{rms}}\left(f_{\mathrm{c}}\right)\right]$, where $h_{\mathrm{rms}}=\sqrt{f S_{h}}$ is the value of the rms strain noise (i.e., the theoretical sensitivity window) for the detector.

\section{NUMERICAL METHODS}

We perform all simulations using the COCONUT code described in detail in $[14,62]$. The equations of general relativistic hydrodynamics are solved in semidiscrete fashion. The spatial discretization is performed by means of a high-resolution shock-capturing scheme employing a second-order accurate finite-volume discretization. We make use of the Harten-Lax-van Leer-Einfeldt (HLLE) flux formula for the local Riemann problems and piecewise-parabolic reconstruction of the primitive variables $\left(\rho, v^{i}, \boldsymbol{\epsilon}\right)$ at cell interfaces. For a review of such methods in general relativistic hydrodynamics, see [66]. The time integration and coupling with curvature are carried out with the method of lines [67] in combination with a second-order accurate explicit Runge-Kutta scheme. Once the state vector $\boldsymbol{U}$ is updated in time, the primitive variables are recovered from the conserved ones given in Eq. (3) through an iterative Newton-Raphson method. Note that 
the component associated to $Y_{e}$ in the system (4) of hydrodynamic evolution equations is treated as a passive advection equation, which does not contribute to the characteristic structure in the form of eigenvalues and eigenvectors required by some flux solvers.

To numerically solve the metric equations we utilize an iterative nonlinear solver based on spectral methods. The spectral grid of the metric solver is split into 6 radial domains with 33 radial and 17 angular collocation points each. The combination of high-resolution shock-capturing methods for the hydrodynamics and spectral methods for the metric equations (the Mariage des Maillages or "grid wedding" approach) in a multidimensional numerical code has been presented in detail in [62]. Even when using spectral methods the calculation of the spacetime metric from the system (12)-(14) of elliptic equations is computationally expensive. Hence, in our simulations the metric is updated only once every 100/10/50 hydrodynamic time steps before/during/after core bounce, and extrapolated in between. The numerical adequacy of this procedure has been tested and discussed in detail in [14].

In this study we focus on the gravitational wave signal associated with core bounce. As demonstrated by [17,25], effects that may break rotational symmetry are most likely unimportant in this context. Hence, we assume axisymmetry and in addition impose symmetry with respect to the equatorial plane.

The COCONUT code utilizes Eulerian spherical coordinates $\{r, \theta\}$, and for the computational grid we choose 250 logarithmically spaced, centrally condensed radial zones with a central resolution of $250 \mathrm{~m}$ and 45 equidistant angular zones covering $90^{\circ}$. A small part of the grid is covered by an artificial low-density atmosphere extending beyond the core's outer boundary defined where $\rho \leq$ $10^{-4} \rho_{\mathrm{c}, \mathrm{i}}$.

We also note that we have performed extensive resolution tests with different grid resolutions to ascertain that the grid setup specified above is appropriate for our simulations.

\section{COLLAPSE DYNAMICS AND WAVEFORM MORPHOLOGY}

\section{A. Generic waveform type}

We begin our discussion with an analysis of the gravitational radiation waveform emitted during core bounce as an indicator for the influence of the EoS, the progenitor structure, and the precollapse rotational configuration on the collapse and bounce dynamics. In Fig. 2, we present example waveforms for representative collapsing cores selected from the investigated parameter space of models (i.e., less or more massive progenitors with slow or rapid precollapse rotation, varying degree of differential rotation, and using either the Shen EoS or LS EoS). The waveforms of all models are of type I, and hence exhibit a positive prebounce rise and a large negative peak, fol-
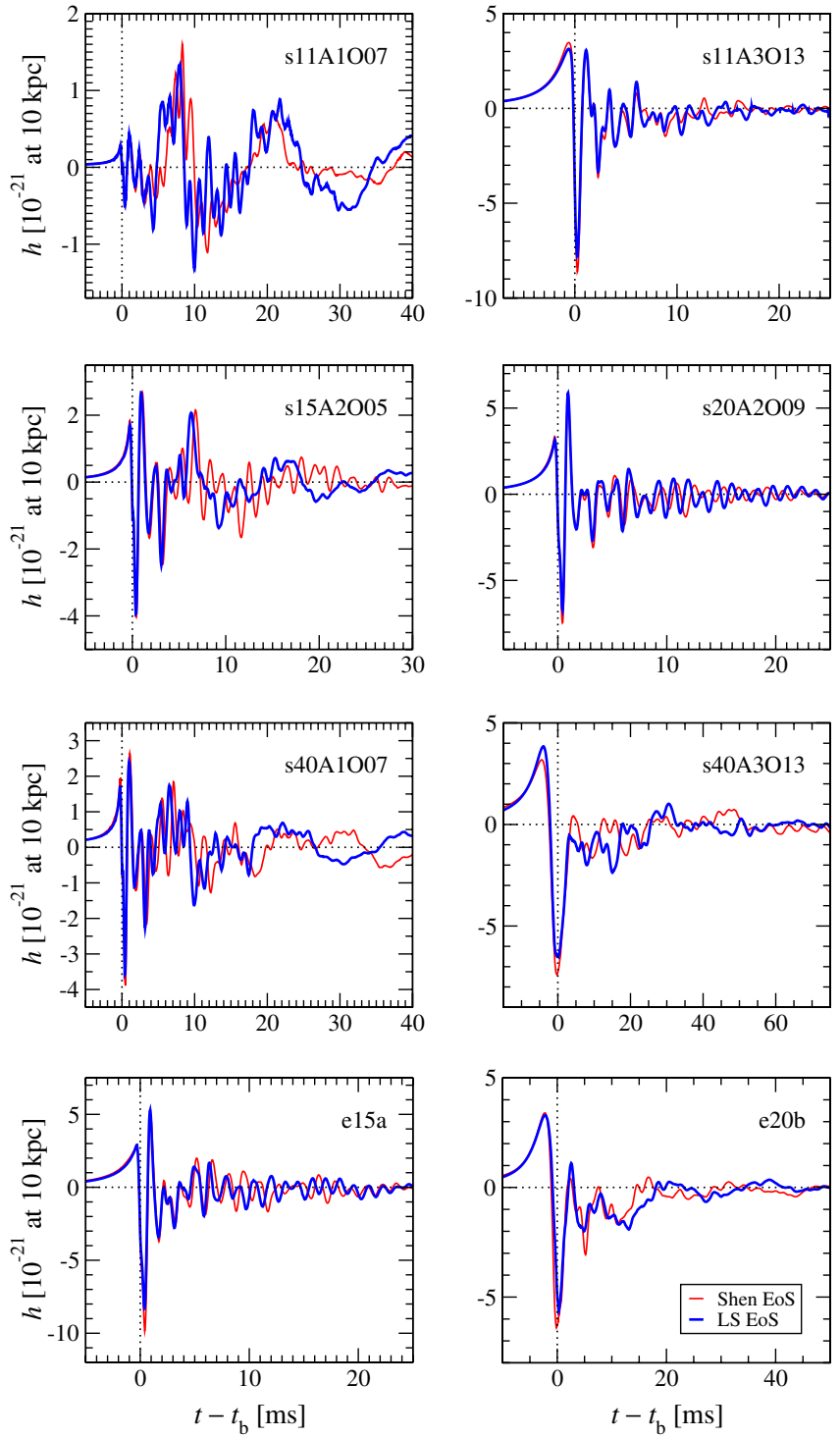

FIG. 2 (color online). Time evolution of the gravitational wave amplitude $h$ for representative models with different precollapse rotation profiles using the Shen EoS (red lines) or LS EoS (blue lines). Models with slow and almost uniform precollapse rotation (e.g., s11A1O07) develop considerable prompt postbounce convection visible as a dominating lower-frequency contribution in the waveform, while the waveform for both models with moderate rotation (e.g., s11A3O13, s15A2O05, s20A2O09, s40A1O07, or e15a) and rapidly rotating models, which undergo a centrifugal bounce (e.g., s40A3013 or e20b), exhibit an essentially regular ringdown. Time is normalized to the time of bounce $t_{\mathrm{b}}$.

lowed by a ringdown. In the light of a considerably extended parameter space in terms of EoS and progenitor mass of the rotating core-collapse models investigated in this work, we thus confirm the observation presented in $[17,18,38,41]$ that in general relativistic gravity all models with microphysics exhibit gravitational wave burst signals of type I. 
As already inferred in $[18,41]$, this signal type can be classified into three subtypes, which, however, do have in common the same qualitative features of a type I waveform described above:

(1) For a slowly rotating core, prompt convective overturn at early postbounce times after the pressuredominated bounce adds a significant low-frequency contribution to the regular ringdown signal (see, e.g., model s11A1O07).

(2) In the case of moderately rapid rotation, which still leads to a pressure-dominated bounce, this convection is effectively suppressed due to the growing influence of angular momentum gradients [33,68] and does not strongly stand out in the postbounce ringdown signal (see, e.g., models s11A3O13, s15A2O05, s20A2O09, s40A1O07, or e15a).

(3) If rotation is sufficiently rapid, the core bounces at subnuclear or only slightly supernuclear densities due to the increased effects of centrifugal forces, reflected by a significant widening of the bounce peak of the waveform and an overall lower fre-

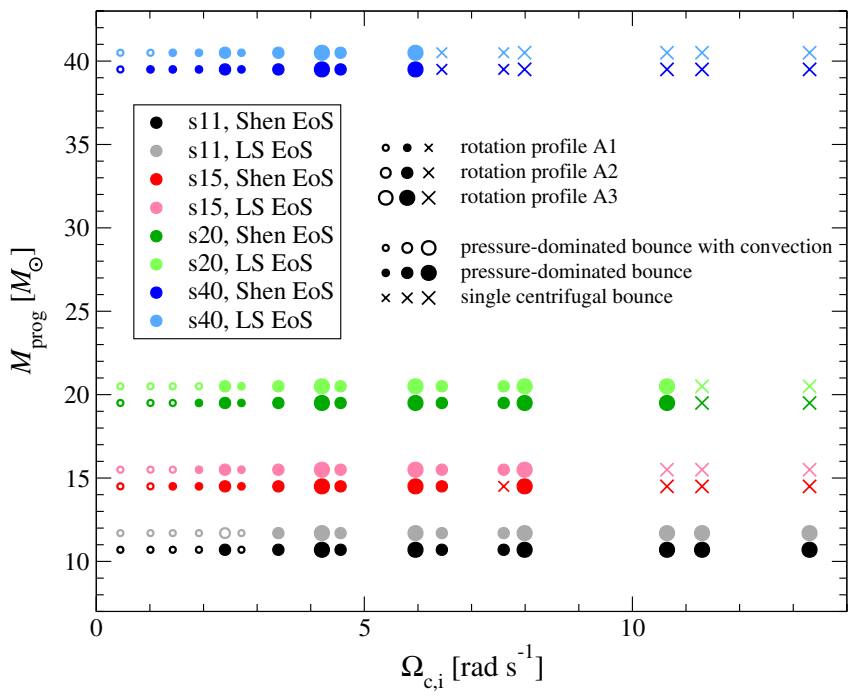

FIG. 3 (color online). Collapse dynamics of all investigated models in the parameter space of precollapse rotational configuration (specified by the precollapse angular velocity $\Omega_{\mathrm{c}, \mathrm{i}}$ at the center and the precollapse differential rotation length scale $A$ ), progenitor mass $M_{\text {prog }}$, and EoS. Models marked by unfilled/ filled circles undergo a pressure-dominated bounce with/without significant early postbounce convection, while models marked with crosses show a single centrifugal bounce. The EoS is encoded as in Fig. 1, while small/medium/large symbols represent the precollapse rotation parameter A1/A2/A3. For better visibility, the symbols for the same $M_{\text {prog }}$ but different EoS are spread a bit in the vertical direction. Note also that in this and the following plots that encode the precollapse rotational configuration in the form of the parameter $\Omega_{i}$, we refrain from including models e15a, e15b, e20a, and e20b, as these have a precollapse rotation profile that is not given by the analytic rotation law (18). quency of the signal (see, e.g., models s40A3O13 or e20b).

Figure 2 also demonstrates that for comparable precollapse rotational configuration (as specified by the parameters $A$ and $\Omega_{\mathrm{i}}$ ) the impact of the EoS on the collapse dynamics and, hence, the gravitational wave signal is small. In Table III, we mark each model with its type of collapse dynamics, and in Fig. 3 we encode that type in the parameter space spanned by rotational configuration, progenitor mass/model, and EoS.

For our set of collapse models, only in four cases (models s11A1O13, s15A1O07, s20A1O09, and s40A1O05) the LS EoS yields a signal with dominant convective contribution, while the Shen EoS does not, and only a single model (s15A2O15) changes its collapse type from a centrifugal bounce to a pressure-dominated bounce when replacing the Shen EoS with the LS EoS. However, Fig. 3 shows that the transition between the three different collapse and waveform subtypes occurs at different precollapse rotational configurations for the various progenitor masses. This is a consequence of differences in the mass $M_{\mathrm{ic}, \mathrm{b}}$ of the inner core at bounce as discussed in Sec. IV C.

The growth of the strong prompt early postbounce convection in slowly rotating models depends sensitively on the seed perturbations resulting from the numerical scheme/grid. In nature, prompt convection will be influenced by random small-scale to large-scale variations in the final stages of silicon burning that are frozen in during collapse. We point out that the duration of the prompt postbounce convection is most likely overestimated in our approach, since in full postbounce radiationhydrodynamics calculations, energy deposition by neutri-

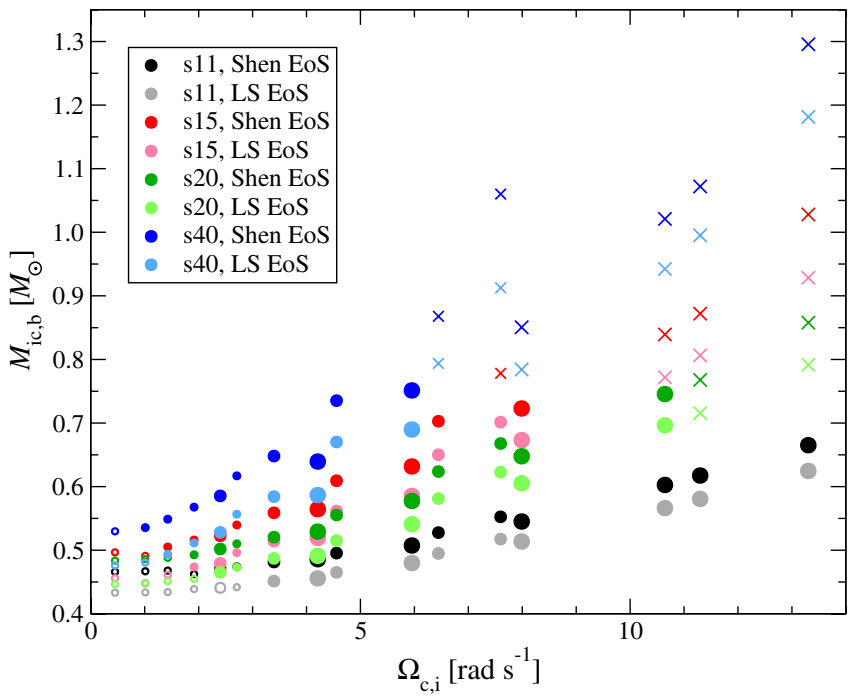

FIG. 4 (color online). Mass $M_{\mathrm{ic}, \mathrm{b}}$ of the inner core at the time of bounce for all models versus the precollapse initial central angular velocity $\Omega_{\mathrm{c}, \mathrm{i}}$. The progenitor model, the EoS, the initial rotation parameter $A$, and the collapse dynamics are encoded as in Fig. 3. 
nos in the immediate postshock region rapidly smooths out the negative entropy gradient left behind by the shock (see, e.g., [20,69]) and quickly damps this early convective instability.

\section{B. Influence of general relativity and deleptonization}

The general type of collapse and bounce dynamics of the core, i.e., pressure-dominated or centrifugal bounce, can be influenced (provided that the description of gravity and neutrino effects are identical) by the progenitor core stratification and thermodynamic structure, the amount and precollapse distribution of angular momentum, and the properties of the EoS in the density regime just below the stiffening threshold [18,41]. These conditions influence the mass $M_{\mathrm{ic}, \mathrm{b}}$ of the homologously contracting inner core at bounce, which in turn determined the region that is dynamically relevant at bounce and sets the initial size of the proto-neutron star.

In Fig. 4, we show the resulting variation of $M_{\mathrm{ic}, \mathrm{b}}$ with $\Omega_{\mathrm{c}, \mathrm{i}}$, progenitor model, precollapse differential rotation parameter $A$, EoS, and collapse type (encoded via symbols as in Fig. 3). The details of the variation of $M_{\mathrm{ic}, \mathrm{b}}$ with progenitor, EoS and rotational configuration will be discussed in Sec. IVC. In the following, without loss of generality, we focus on a single progenitor and discuss the influence of general relativity and deleptonization on the collapse dynamics and the gravitational wave burst signal along the lines of the discussion in $[18,41]$.

In order to assess the individual influence of relativistic effects and deleptonization, and to explain the absence of type II and III burst signals in microphysical general relativistic models, in $[18,41]$ we compared collapse models of the s20 progenitor using the Shen EoS and a description for deleptonization with models using a simple hybrid polytropic/ $\gamma$-law EoS. We selected the adiabatic index $\gamma_{\text {eos }}$ of these simple models in such a way that the transition between pressure-dominated bounce and centrifugal bounce occurs at the same precollapse rotation rate $\beta_{\mathrm{i}}$ as for the microphysical models. With this method we were able to demonstrate that the influence of deleptonization can be approximated by a correction $\Delta \gamma_{\nu} \simeq 0.03$ that must be applied to the estimate of the average EoS adiabatic index $\gamma_{\text {eos,Shen }} \simeq 1.32$ in the density interval between $10^{12}$ and $10^{14} \mathrm{~g} \mathrm{~cm}^{-3}$. This leads to a generic value for the effective adiabatic index $\gamma_{\text {eff,Shen }}=\gamma_{\text {eos,Shen }}-\Delta \gamma_{\nu} \simeq$ $1.32-0.03=1.29$, practically independent of the precollapse rotational configuration, both in Newtonian gravity and general relativity (where relativistic effects are accounted for by a correction $\left.\Delta \gamma_{\mathrm{gr}} \simeq-0.015\right)$. A graphic representation of this argument is shown in the top panel of Fig. 5, that is identical to Fig. 2 in [18] and Fig. 4 in [41], and which we include here for completeness.

The estimate $\gamma_{\text {eff }} \simeq 1.29$ for microphysical models also allows us to explain the suppression of multiple centrifugal bounces with an associated type II waveform in a straight-
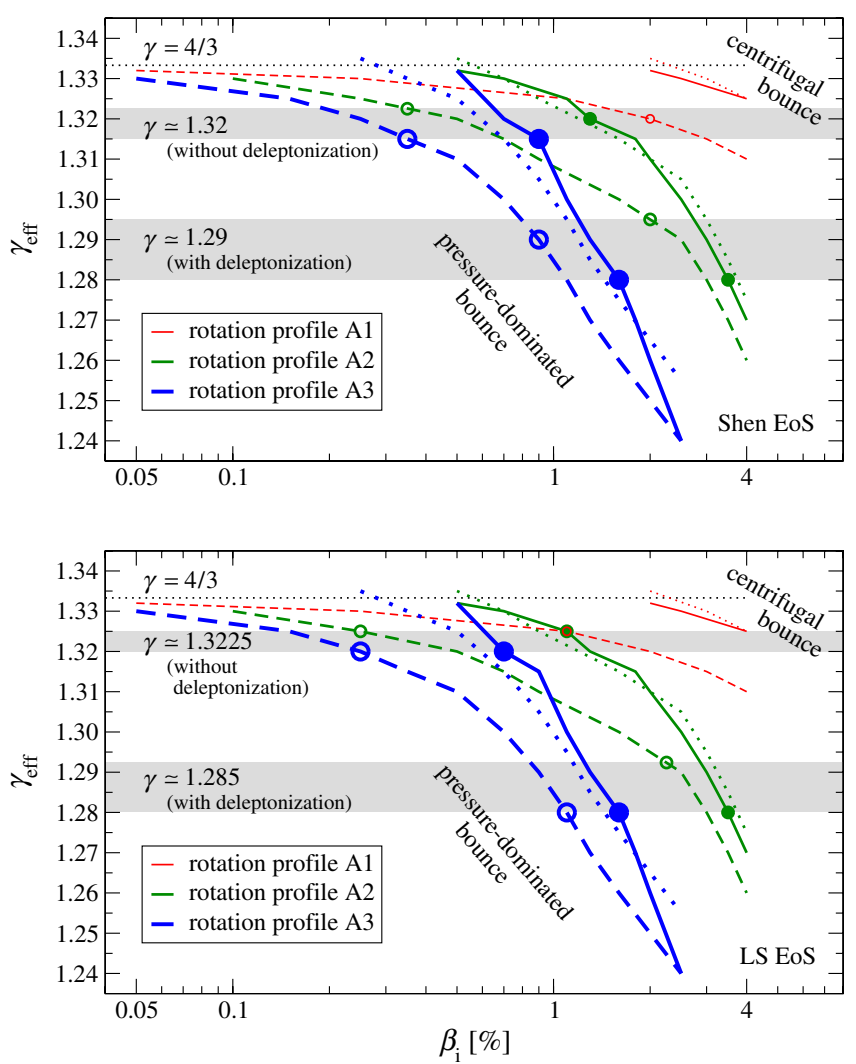

FIG. 5 (color online). Boundary between pressure-dominated and centrifugal bounce in the $\gamma_{\mathrm{eff}}-\beta_{\mathrm{i}}$ plane for s20 progenitor models using the hybrid EoS in Newtonian gravity (dashed lines) and general relativity (solid lines). The curved dotted lines show the Newtonian results shifted by $-\Delta \gamma_{\mathrm{gr}}=0.015$. The transition points for models using the microphysical EoS without and with deleptonization, again for Newtonian gravity (circles) and general relativity (bullets), lie in the shaded areas around $\gamma_{\mathrm{eos} \text {, Shen }} \simeq$ 1.32 and $\gamma_{\mathrm{eff}, \text { Shen }} \simeq 1.29$, respectively, for the Shen EoS (top panel) and around $\gamma_{\mathrm{eos}, \mathrm{LS}} \simeq 1.3225$ and $\gamma_{\mathrm{eff}, \mathrm{LS}} \simeq 1.285$, respectively, for the LS EoS (bottom panel).

forward way, since this type of collapse occurs only in the respective hybrid EoS models with an effective adiabatic index that is much closer to $4 / 3$, i.e., $\gamma_{\text {eff }} \geq 1.31$. Rapid collapse dynamics that is characterized by a type III burst signal is also not realized in microphysical models of massive star collapse, as it requires a mass of the inner core at bounce $M_{\text {ic,b }} \lesssim 0.3 M_{\odot}$ [13], which is considerably smaller than those found in microphysical models with any of our progenitors, for which we find $M_{\text {ic }} \gtrsim 0.4 M_{\odot}$ (see Fig. 4, and also the discussion in Sec. IV C). However, in $[25,70]$ it is suggested that rapid collapse dynamics and a type III burst signal may be associated with very efficient electron capture in the accretion-induced collapse of massive, rapidly rotating white dwarfs.

Finally, we point out that calculations with $\gamma_{\text {eff }}$ used in the hybrid EoS have the tendency to underestimate the mass $M_{\mathrm{ic}, \mathrm{b}}$ at bounce compared with the full microphysical treatment. This is a consequence of the fact that in these 
calculations $\gamma_{\text {eff }}$ is kept constant throughout the collapse, leading to a reduction of the inner core mass $M_{\mathrm{ic}}$ already at much earlier collapse stages than in microphysical models. The underestimated $M_{\mathrm{ic}, \mathrm{b}}$, in turn, leads to gravitational wave burst signals from bounce in those simple models that are quantitatively or even qualitatively incorrect (as in the case of type III signals, which do not occur in microphysical models). Hence, while useful for understanding the collapse dynamics, the $\gamma_{\text {eff }}$ approach cannot replace the full microphysical treatment with a nonzero-temperature microphysical EoS and deleptonization as performed in the present work.

\section{Influence of the equation of state and progenitor model}

At densities below $\rho_{\text {nuc }}$ the total fluid pressure is dominated by the contribution from the degenerate electrons, hence the two microphysical EoSs should lead to rather similar dynamics in the infall phase of collapse. This is also reflected in the very similar behavior of their adiabatic indices $\gamma_{\text {eos }}$ as shown in Fig. 6.

In the bottom panel of Fig. 5, we demonstrate that the same influence of general relativistic effects and deleptonization as discussed in Sec. IV B applies for the s20 progenitor when the LS EoS is used instead of the Shen EoS. We obtain values of $\gamma_{\text {eos,LS }} \simeq 1.3225$ for the adiabatic index of the EoS (without deleptonization) and $\gamma_{\text {eff,LS }} \simeq$ 1.285 for the effective adiabatic index (including deleptonization), which is in very close agreement with the values deduced from the simulations using the Shen EoS. As

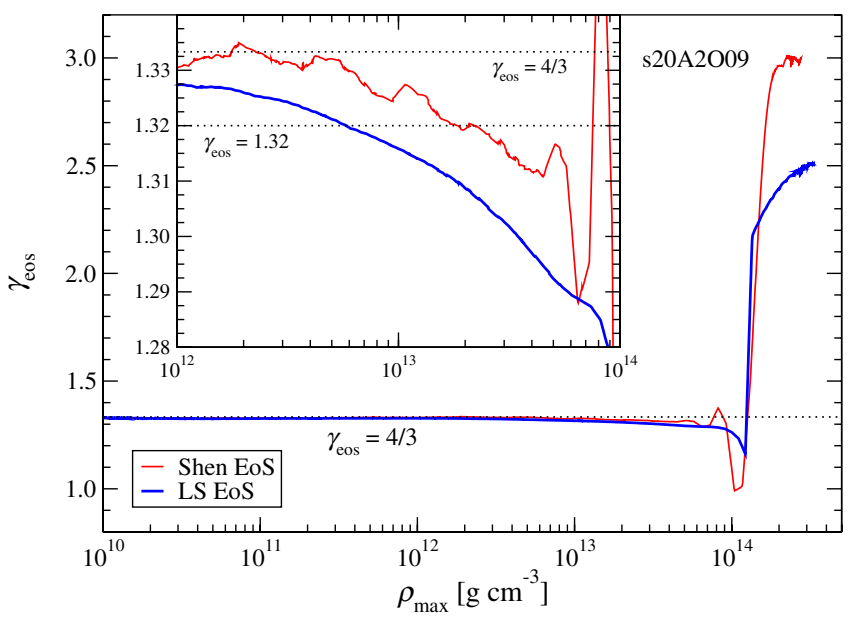

FIG. 6 (color online). Adiabatic index $\gamma_{\mathrm{eos}}$ of the Shen EoS (red line) and LS EoS (blue line) versus the maximum density $\rho_{\max }$ in the collapsing core for model s20A2O09. Although $\rho_{\max }$, which for this model is located in the center of the core, does not follow a trajectory of constant specific entropy, $s$ is still approximately conserved in the prebounce phase. Inset: Magnified view of $\gamma_{\mathrm{eos}}$ in the dynamically most relevant density range between $10^{12}$ and $10^{14} \mathrm{~g} \mathrm{~cm}^{-3}$. The average value of $\gamma_{\mathrm{eos}}$ in this density regime is roughly 1.32 for both EoSs. shown in Fig. 7, now only for general relativistic gravity, there is some spread of $\gamma_{\mathrm{eff}}$ with progenitor mass/model, but on average, we find $\gamma_{\mathrm{eff}} \simeq 1.28$ for the $\mathrm{s} 11, \mathrm{~s} 15$, and $\mathrm{s} 40$ progenitor models.

Again following the line of arguments presented in $[18,41]$, the combination of a low effective adiabatic index $\gamma_{\text {eff }}<1.31$ and a high inner core mass $M_{\text {ic }} \gtrsim 0.4 M_{\odot}$ at bounce results in a type I gravitational wave burst signal for all our models, independent of the EoS or progenitor model. Note that creating Figs. 5 and 7 we have performed additional simulations of microphysical models that are more narrowly spaced in $\Omega_{\mathrm{i}, \mathrm{c}}$ (and correspondingly in $\beta_{\mathrm{i}}$ ) than the ones listed in Table II. As a result, those figures reveal a small dependence of the transition between pressure-dominated bounce and centrifugal bounce (i.e., the location of the bullets and circles in the direction of the abscissa) on the EoS, which is generally not apparent from Table II.

Although the sensitivity of the deleptonization and collapse dynamics on the progenitor mass and EoS is only small, Fig. 4 still reveals a dependence of the inner core mass $M_{\mathrm{ic}, \mathrm{b}}$ at bounce both on the EoS and (in particular) on rotation. Furthermore, $M_{\mathrm{ic}, \mathrm{b}}$ varies nonmonotonically with the progenitor mass $M_{\text {prog }}$. In the absence of rotation, $M_{\mathrm{ic}, \mathrm{b}}$ is solely determined by the mean trapped lepton fraction $Y_{\text {lep }}=Y_{e}+Y_{\nu}$ and specific entropy $s$ in the inner core $[1,2,27,71]$ with a roughly quadratic dependence on both quantities. Since we employ the same $Y_{e}(\rho)$ parametrization (based on model s20) for all models with the same

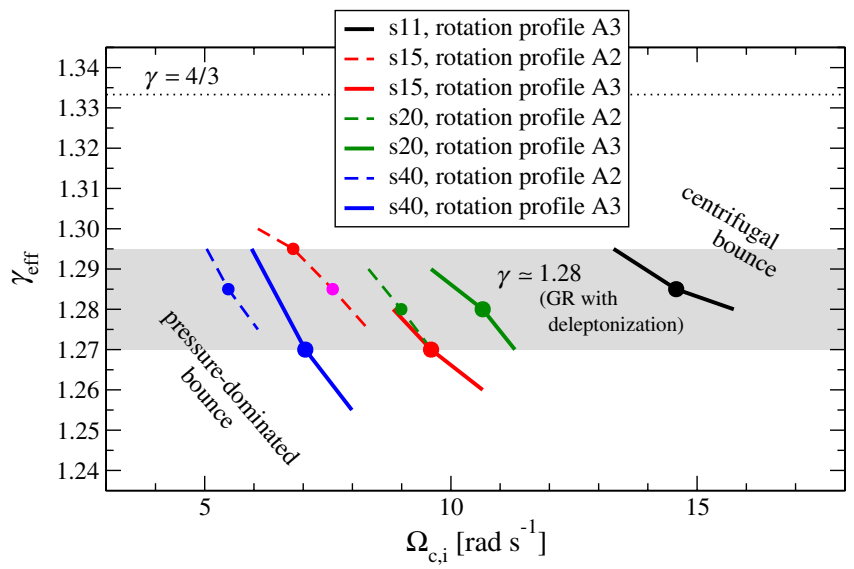

FIG. 7 (color online). Boundary between pressure-dominated and centrifugal bounce in the $\gamma_{\mathrm{eff}}-\Omega_{\mathrm{c}, \mathrm{i}}$ plane for models of all progenitors using the hybrid EoS in general relativity. The transition points for models using the microphysical EoS with deleptonization (bullets) lie in the shaded area around $\gamma_{\mathrm{eff}} \simeq$ 1.28. Except for the rotation profile A2 of the s15 progenitor, the locations of these points are identical for the two EoSs. Note that for the A1 profiles of any progenitor (and the A 2 profile for the s11 progenitor) we do not observe a centrifugal bounce for any value of $\Omega_{\mathrm{c}, \mathrm{i}}$. In contrast to Fig. 5, we use here the $\Omega_{\mathrm{c}, \mathrm{i}}$ instead of $\beta_{\mathrm{i}}$ as parameter for the precollapse rotational configuration (see the discussion in Sec. IIE). 
EoS, the variations in $M_{\mathrm{ic}, \mathrm{b}}$ are caused by differences in the specific entropy in the precollapse iron core. Generally, the specific entropy in the iron core increases with progenitor mass, but, in particular, in the mass range of $\sim 18-25 M_{\odot}$, the relationship of progenitor mass and specific core entropy can be nonmonotonic (see, e.g., [58]). However, note that the systematics for $M_{\mathrm{ic}, \mathrm{b}}$ with progenitor mass seen in Fig. 4 are possibly overemphasized by our $Y_{e}(\rho)$ parametrization and may be less pronounced in full radiation transport simulations, which remain to be carried out in the future.

For a rotating collapse, the variations of $M_{\mathrm{ic}, \mathrm{b}}$ with progenitor mass are amplified, while the overall systematics are preserved. Obviously, a more massive and hence more extended inner core is more susceptible to the influence of centrifugal forces (which scale proportional to the radius $r$ ) than a less massive and thus more compact inner core. This behavior is confirmed by Fig. 4, which depicts the dependence of the mass $M_{\mathrm{ic}, \mathrm{b}}$ of the inner core at bounce on the precollapse central angular velocity $\Omega_{\mathrm{c}, \mathrm{i}}$, the EoS, and the differential rotation parameter $A$. Models with comparably large precollapse iron core specific entropy (and also large iron core mass) and, thus, larger $M_{\mathrm{ic,b}}$ already in the nonrotating case, show a more pronounced increase of $M_{\mathrm{ic}, \mathrm{b}}$ with rotation than models with lower precollapse specific entropy (and also smaller iron core mass). The scaling of $M_{\mathrm{ic}, \mathrm{b}}$ with $\Omega_{\mathrm{c}, \mathrm{i}}$, at fixed differential rotation parameter $A$, is linear for small to intermediate $\Omega_{\mathrm{c}, \mathrm{i}}$ and turns approximately quadratic for the most rapidly rotating configurations. On the other hand, when increasing the degree of differential rotation $A$ at fixed $\Omega_{\mathrm{c}, \mathrm{i}}, M_{\mathrm{ic}, \mathrm{b}}$ decreases since then the angular velocity in the outer parts of the inner core and consequently centrifugal support drops.

We also observe that the impact of the EoS on the mass of the inner core manifests itself only via an almost constant positive relative increase in $M_{\mathrm{ic}, \mathrm{b}}$ of $\sim 10 \%$ when changing from the LS EoS to the Shen EoS, practically independent of rotation and progenitor mass (see Fig. 4). Again, the mean electron (respectively lepton) fraction and specific entropy in the inner core are the key to understanding these systematics. The representative s20 progenitor model used to parametrize $Y_{e}(\rho)$ in this study yields minima for $Y_{e}$ in the center of the core at bounce of $\sim 0.249$ and $\sim 0.241$ for the Shen EoS and the LS EoS, respectively. This relative difference of $\sim 3.3 \%$ translates into a difference in $M_{\mathrm{ic}, \mathrm{b}}$ of $\sim 7 \%$, assuming that the mass of the inner core scales quadratically with $Y_{e}$, which slightly underestimates the actual change. We attribute the remaining difference to variations in the specific entropy $s$ of the inner core at bounce due to the slightly more efficient electron capture in the models with the LS EoS.

We point out that the progenitor models e15a, e15b, $\mathrm{e} 20 \mathrm{a}$, and $\mathrm{e} 20 \mathrm{~b}$, which already come with a rotation profile from the stellar evolution calculation, are very well represented in terms of collapse dynamics, waveform, and postbounce rotation state by members of our model set with an artificially added precollapse rotation profile, specifically the models s15A2O09, s15A2O15, s20A2O09, and s20A2O15. For this reason we refrain from separately discussing those special models in this paper.

\section{Influence of differential rotation}

Increasing the degree of differential rotation by lowering the value of the differential rotation parameter $A$ at fixed precollapse central angular velocity $\Omega_{\mathrm{c}, \mathrm{i}}$ results in less centrifugal support in outer core regions and, as already pointed out in Sec. IVC, in a smaller mass $M_{\mathrm{ic}, \mathrm{b}}$ of the inner core at bounce. Consequently, a higher value of $\Omega_{c, i}$ is necessary for a stronger differentially rotating precollapse core to become significantly affected by centrifugal forces during the collapse. This is confirmed by Fig. 7, which displays the systematics of the transition between pressure-dominated and centrifugal bounce for our set of progenitors and the $\mathrm{A} 2$ and $\mathrm{A} 3$ rotation profiles. Compared with the transition values of $\Omega_{\mathrm{c}, \mathrm{i}}$ for the A2 profile, the A3 profile requires a roughly $20-40 \%$ higher $\Omega_{\mathrm{c}, \mathrm{i}}$ (varying slightly with progenitor model) for a transition from pressure-dominated to centrifugal bounce.

In previous extensive parameter studies of rotating stellar core collapse (see, e.g., [14-16]) the effect of differential rotation was studied in model sequences in the parameter space spanned by the precollapse differential rotation parameter $A$ and the precollapse rotation rate $\beta_{\mathrm{i}}$. At a constant $\beta_{\mathrm{i}}$, more differentially rotating models require a larger $\Omega_{\mathrm{c}, \mathrm{i}}$ than less differentially rotating ones and experience core bounce at lower densities. Hence, at fixed $\beta_{\mathrm{i}}$, more differentially rotating models are generally more affected by centrifugal effects. Our s20 model series is constructed as a sequence of fixed $\beta_{\mathrm{i}}$ for each of the rotation profiles $\mathrm{A} 1, \mathrm{~A} 2$, and $\mathrm{A} 3$ (see Table II), and therefore permits a direct comparison with preceding work. Our results confirm qualitatively the previously identified systematics (see Table III). However, in contrast to more simplistic simulations, the combination of general relativity and deleptonization in our models weakens the overall impact of centrifugal effects on the collapse dynamics (see Sec. IV B), and consequently leads to much smaller quantitative changes in the characteristic collapse variables (such as $\rho_{\mathrm{max}, \mathrm{b}},|h|_{\max }, M_{\mathrm{ic}, \mathrm{b}}$, or $\beta_{\mathrm{b}}$ ) when varying the degree of differential rotation.

\section{STRUCTURE OF THE POSTBOUNCE CORE AND IMPACT ON THE WAVE SIGNAL}

\section{A. Equation of state at supernuclear densities and maximum density in the core}

From Table III it is apparent that the change from the Shen EoS to the LS EoS in an otherwise identical model 
TABLE III. Summary of relevant quantities from the rotating collapse of all iron core models. $\rho_{\text {max,b }}$ is the maximum density in the core at the time of bounce, $|h|_{\max }$ is the peak value of the gravitational wave amplitude for the burst signal, while $\beta_{\mathrm{b}}$ and $\beta_{\mathrm{pb}}$ are the rotation rates at the time of bounce and late after core bounce, respectively. Models marked by unfilled/filled circles undergo a pressure-dominated bounce with/without significant early postbounce convection, while models marked with crosses show a single centrifugal bounce. The values left/right of the vertical separator (l) are for the Shen/LS EoS.

\begin{tabular}{|c|c|c|c|c|c|c|c|c|c|c|c|}
\hline $\begin{array}{l}\text { Collapse } \\
\text { model }\end{array}$ & & $\begin{array}{c}\rho_{\max , \mathrm{b}} \\
{\left[\frac{10^{14}}{\mathrm{~g} \mathrm{~cm}^{-3}}\right]}\end{array}$ & $\begin{array}{c}|h|_{\max } \\
{\left[10^{-21} \text { at } 10 \mathrm{kpc}\right]}\end{array}$ & $\begin{array}{c}\beta_{\mathrm{b}} \\
{[\%]}\end{array}$ & $\begin{array}{l}\beta_{\mathrm{pb}} \\
{[\%]}\end{array}$ & $\begin{array}{c}\text { Collapse } \\
\text { model }\end{array}$ & & $\begin{array}{c}\rho_{\max , \mathrm{b}} \\
{\left[\frac{10^{1 /}}{\mathrm{g} \mathrm{cm}^{-3}}\right]}\end{array}$ & $\begin{array}{c}|h|_{\max } \\
{\left[10^{-21} \text { at } 10 \mathrm{kpc}\right]}\end{array}$ & $\begin{array}{c}\beta_{\mathrm{b}} \\
{[\%]}\end{array}$ & $\begin{array}{l}\beta_{\mathrm{pb}} \\
{[\%]}\end{array}$ \\
\hline s11A1O01 & 이잉 & $3.24 \mid 4.43$ & $0.05 \mid 0.05$ & $0.1 \mid 0.1$ & $0.1 \mid 0.1$ & s15A1O01 & 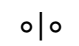 & $3.28 \mid 4.43$ & $0.20 \mid 0.20$ & $0.2 \mid 0.2$ & $0.3 \mid 0.3$ \\
\hline s11A1O05 & 이잉 & $3.23 \mid 4.41$ & $0.26 \mid 0.25$ & $.3 \mid 0.3$ & $0.4 \mid 0.5$ & s15A1O05 & 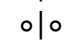 & $3.17 \mid 4.20$ & $0.98 \mid 0.97$ & $1.0 \mid 1.0$ & $1.3 \mid 1.2$ \\
\hline s11A1O07 & 이잉 & $3.22 \mid 4.35$ & $0.51 \mid 0.49$ & $0.6 \mid 0.6$ & $0.8 \mid 0.8$ & s15A1O07 & $\bullet 10$ & $3.12 \mid 4.13$ & $1.84 \mid 1.84$ & $2.0 \mid 1.9$ & $2.4 \mid 2.6$ \\
\hline s11A1O09 & 이잉 & $3.17 \mid 4.21$ & $0.95 \mid 0.90$ & $1.1 \mid 1.1$ & $1.3 \mid 1.4$ & s15A1O09 & $\bullet \mid \bullet$ & $2.97 \mid 3.88$ & $3.11 \mid 3.08$ & $3.4 \mid 3.4$ & $3.7 \mid 4.0$ \\
\hline s11A1O13 & - 10 & $3.11 \mid 4.13$ & $1.77 \mid 1.76$ & $2.0 \mid 2.0$ & $2.4 \mid 2.6$ & s15A1O13 & $\bullet \mid \bullet$ & $2.86 \mid 3.56$ & $5.35 \mid 5.01$ & $6.2 \mid 6.1$ & $6.0 \mid 6.6$ \\
\hline s11A2O05 & 이잉 & $3.16 \mid 4.18$ & $1.30 \mid 1.35$ & $1.4 \mid 1.5$ & $1.6 \mid 1.7$ & s15A2O05 & $\bullet \mid \bullet$ & $2.95 \mid 3.76$ & $4.04 \mid 3.94$ & 4.1|4.1 & $3.8 \mid 4.3$ \\
\hline s11A2O07 & $\bullet \mid \bullet$ & $3.02 \mid 3.92$ & $2.47 \mid 2.52$ & $2.8 \mid 2.8$ & $2.9 \mid 3.0$ & s15A2O07 & $\bullet \mid \bullet$ & $2.81 \mid 3.44$ & $6.84 \mid 6.33$ & $7.5 \mid 7.4$ & $6.7 \mid 6.8$ \\
\hline s11A2O09 & $\bullet \mid \bullet$ & $2.94 \mid 3.69$ & $4.08 \mid 4.00$ & $4.8 \mid 4.8$ & $4.6 \mid 4.7$ & s15A2O09 & $\bullet \mid \bullet$ & $2.58 \mid 3.05$ & $8.61 \mid 7.83$ & $11.8 \mid 11.6$ & $10.3 \mid 10.4$ \\
\hline $\mathrm{s} 11 \mathrm{~A} 2 \mathrm{O} 13$ & $\bullet \mid \bullet$ & $2.76 \mid 3.35$ & $6.68 \mid 6.09$ & $8.5 \mid 8.4$ & $7.9 \mid 8.0$ & $\mathrm{~s} 15 \mathrm{~A} 2 \mathrm{O} 13$ & $\bullet \mid \bullet$ & $2.14 \mid 2.33$ & $7.07 \mid 6.21$ & $18.2 \mid 17.5$ & $15.6 \mid 15.5$ \\
\hline s11A2O15 & $\bullet \mid \bullet$ & $2.66 \mid 3.15$ & $7.72 \mid 7.01$ & $10.9 \mid 10.8$ & $9.8 \mid 9.9$ & s15A2O15 & $\times 1 \bullet$ & $1.80 \mid 1.90$ & $4.01 \mid$ & $20.1 \mid 19.7$ & $17.9 \mid 18.1$ \\
\hline s11A3O05 & $\bullet \mid \bullet$ & $3.02 \mid 3.88$ & $2.96 \mid 3.05$ & $3.2 \mid 3.2$ & $2.8 \mid 3.0$ & s15A3O05 & $\bullet \mid \bullet$ & $2.82 \mid 3.46$ & $7.65 \mid 7.27$ & $7.2 \mid 7.4$ & $5.7 \mid 5.8$ \\
\hline s11A3O07 & $\bullet \mid \bullet$ & $2.89 \mid 3.60$ & $5.33 \mid 5.30$ & $5.9 \mid 6.0$ & $5.1 \mid 5.2$ & s15A3O07 & $\bullet \mid \bullet$ & $2.55 \mid 2.94$ & $10.06 \mid 9.55$ & $12.8 \mid 12.7$ & $10.0 \mid 9.9$ \\
\hline s11A3O09 & $\bullet \mid \bullet$ & $2.71 \mid 3.25$ & $8.42 \mid 7.66$ & $9.7 \mid 9.7$ & $8.0 \mid 8.2$ & s15A3O09 & $\bullet \mid \bullet$ & $2.17 \mid 2.31$ & 9.74 & $18.7 \mid 18.1$ & $14.8 \mid 14.6$ \\
\hline s11A3O12 & $\bullet \mid \bullet$ & $2.46 \mid 2.75$ & $8.92 \mid 7.84$ & $14.9 \mid 14.7$ & $12.3 \mid 12.3$ & s15A3O12 & $x \mid x$ & $1.15 \mid 1.26$ & $5.68 \mid 4.82$ & $21.1 \mid 21.0$ & $18.3 \mid 18.9$ \\
\hline s11A3O13 & $\bullet \mid \bullet$ & $2.36 \mid 2.64$ & $8.62 \mid 7.73$ & $16.1 \mid 15.8$ & $13.2 \mid 13.2$ & s15A3O13 & $\times \mid x$ & $0.72 \mid 0.84$ & 5.33 & $21.3 \mid 21.3$ & $18.9 \mid 19.6$ \\
\hline s11A3O15 & $\bullet \mid \bullet$ & $2.10 \mid 2.23$ & $7.21 \mid 6.32$ & $19.4 \mid 18.6$ & $16.2 \mid 15.8$ & s15A3O15 & $x \mid x$ & $0.25 \mid 0.30$ & 4.84 & $22.2 \mid 22.3$ & $20.3 \mid 21.1$ \\
\hline s20A1O01 & 이잉 & $3.28 \mid 4.41$ & $0.13 \mid 0.13$ & $0.1 \mid 0.1$ & $0.2 \mid 0.2$ & s40A1O01 & 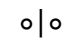 & $3.29 \mid 4.42$ & 0.50 & $0.4 \mid 0.4$ & $0.5 \mid 0.6$ \\
\hline s20A1O05 & 이잉 & $3.21 \mid 4.23$ & $0.63 \mid 0.64$ & $0.7 \mid 0.7$ & $0.9 \mid 1.0$ & s40A1O05 & $\bullet 10$ & $3.13 \mid 4.14$ & 2.12 & $1.9 \mid 1.8$ & $2.1 \mid 2.3$ \\
\hline s20A1O07 & 이잉 & $3.17 \mid 4.23$ & $1.19 \mid 1.28$ & $1.3 \mid 1.3$ & $1.6 \mid 1.9$ & s40A1O07 & $\bullet \mid \bullet$ & $2.96 \mid 3.89$ & 3.89 & $3.5 \mid 3.5$ & $3.7 \mid 4.5$ \\
\hline s20A1O09 & - 10 & $3.10 \mid 4.11$ & $2.20 \mid 2.12$ & $2.3 \mid 2.3$ & $2.6 \mid 3.0$ & s40A1O09 & $\bullet \mid \bullet$ & $2.85 \mid 3.64$ & $5.97 \mid$ & $5.9 \mid 5.8$ & $5.7 \mid 6.5$ \\
\hline s20A1O13 & $\bullet \mid \bullet$ & $2.95 \mid 3.77$ & $3.81 \mid 3.68$ & $4.3 \mid 4.3$ & $4.5 \mid 5.0$ & s40A1O13 & $\bullet \mid \bullet$ & $2.63 \mid 3.22$ & $8.30 \mid 7.07$ & $10.2 \mid 9.9$ & $9.4 \mid 9.5$ \\
\hline s20A2O05 & $\bullet \mid \bullet$ & $3.03 \mid 3.94$ & $2.89 \mid 2.89$ & $3.0 \mid 3.0$ & $2.9 \mid 3.1$ & s40A2O05 & $\bullet \mid \bullet$ & $2.81 \mid 3.57$ & $7.43 \mid$ & $6.8 \mid 6.7$ & $5.7 \mid 5.8$ \\
\hline s20A2O07 & $\bullet \mid \bullet$ & $2.90 \mid 3.63$ & $5.04 \mid 4.87$ & $5.5 \mid 5.5$ & $5.1 \mid 5.3$ & s40A2O07 & $\bullet \mid \bullet$ & $2.57 \mid 3.06$ & 9.95 & $11.8 \mid 11.6$ & $9.9 \mid 9.9$ \\
\hline s20A2O09 & $\bullet \mid \bullet$ & $2.75 \mid 3.31$ & $7.46 \mid 6.73$ & $9.0 \mid 8.8$ & $8.0 \mid 8.3$ & s40A2O09 & $\bullet \mid \bullet$ & $2.22 \mid 2.44$ & $9.22 \mid 7.80$ & $17.3 \mid 16.7$ & $14.3 \mid 14.3$ \\
\hline s20A2O13 & $\bullet \mid \bullet$ & $2.42 \mid 2.75$ & $7.83 \mid 7.07$ & $14.8 \mid 14.4$ & $12.8 \mid 12.9$ & s40A2O13 & $x \mid x$ & $0.91 \mid 1.28$ & $4.04 \mid 3.30$ & $20.4 \mid 20.6$ & $19.2 \mid 19.0$ \\
\hline s20A2O15 & $\bullet \mid \bullet$ & $2.20 \mid 2.37$ & $7.00 \mid 6.10$ & $17.8 \mid 17.2$ & $15.4 \mid 15.2$ & s40A2O15 & $x \mid x$ & $0.27 \mid 0.40$ & $3.51 \mid 3.51$ & $21.1 \mid 21.4$ & $20.3 \mid 21.0$ \\
\hline s20A3O05 & $\bullet \mid \bullet$ & $2.92 \mid 3.62$ & $5.59 \mid 5.53$ & $5.6 \mid 5.7$ & $4.6 \mid 4.7$ & s40A3O05 & $\bullet \mid \bullet$ & $2.65 \mid 3.21$ & $10.19 \mid 10.07$ & $10.5 \mid 10.6$ & $7.6 \mid 7.7$ \\
\hline s20A3O07 & $\bullet \mid \bullet$ & $2.70 \mid 3.20$ & $9.50 \mid 8.72$ & $10.1 \mid 10.2$ & $8.0 \mid 8.1$ & s40A3O07 & $\bullet \mid \bullet$ & $2.21 \mid 2.47$ & $10.29 \mid 10.09$ & $17.2 \mid 16.8$ & $12.7 \mid 12.5$ \\
\hline s20A3009 & $\bullet \mid \bullet$ & $2.38 \mid 2.63$ & $9.67 \mid 8.65$ & $15.7 \mid 15.4$ & $12.5 \mid 12.4$ & s40A3O09 & $x \mid x$ & $1.69 \mid 1.72$ & $7.45 \mid 7.72$ & $21.6 \mid 21.4$ & $16.9 \mid 16.8$ \\
\hline s20A3O12 & $\bullet \mid \bullet$ & $1.93 \mid 2.00$ & $6.52 \mid 5.98$ & $21.0 \mid 20.3$ & $17.6 \mid 17.1$ & s40A3O12 & $x \mid x$ & $0.33 \mid 0.40$ & $7.36 \mid 6.34$ & $22.5 \mid 22.8$ & $19.2 \mid 20.1$ \\
\hline s20A3O13 & $x \mid x$ & $1.77 \mid 1.79$ & $5.35 \mid 4.98$ & $21.3 \mid 20.8$ & $18.1 \mid 18.0$ & s40A3O13 & $x \mid \times$ & $0.23 \mid 0.28$ & $7.40 \mid 6.51$ & $22.9 \mid 23.4$ & $19.8 \mid 20.7$ \\
\hline s20A3O15 & $x \mid \times$ & $0.65 \mid 0.75$ & $4.62 \mid 3.78$ & $21.6 \mid 21.5$ & $19.7 \mid 20.2$ & s40A3O15 & $x \mid x$ & $0.09 \mid 0.11$ & $6.90 \mid 6.90$ & $24.4 \mid 25.1$ & $21.5 \mid 22.4$ \\
\hline e15a & $\bullet \mid \bullet$ & $2.66 \mid 3.25$ & $9.85 \mid 8.30$ & $9.7 \mid 9.5$ & $7.6 \mid 7.8$ & & & & & & \\
\hline $\mathrm{e} 15 \mathrm{~b}$ & $x \mid x$ & $1.61 \mid 1.69$ & $3.62 \mid 3.57$ & $20.2 \mid 20.1$ & $18.0 \mid 19.0$ & & & & & & \\
\hline e20a & $\bullet \mid \bullet$ & $2.69 \mid 3.35$ & $9.41 \mid 8.09$ & $8.7 \mid 8.5$ & $6.4 \mid 6.4$ & & & & & & \\
\hline$e 20 b$ & $x \mid \times$ & $1.41 \mid 1.50$ & $6.40 \mid 5.54$ & $21.0 \mid 20.4$ & $18.3 \mid 17.4$ & & & & & & \\
\hline
\end{tabular}

results systematically in an increase of the peak maximum density $\rho_{\max , \mathrm{b}}$ at bounce, i.e., $\rho_{\max , \mathrm{b}, \mathrm{LS}}>\rho_{\max , \mathrm{b}, \text { Shen }}$. This result is in agreement with the previous Newtonian study of Kotake et al. [32] who compared simulations of a single model carried out with the Shen EoS and the LS EoS.

For centrifugally bouncing models, which only marginally exceed or even remain below $\rho_{\text {nuc }}$ at bounce, the absolute increase in the maximum core density at bounce is small, exhibiting a maximum $\Delta \rho_{\text {max }, \mathrm{b}}=\rho_{\text {max }, \mathrm{b}, \mathrm{LS}}-$ $\rho_{\text {max, b, Shen }}=0.13 \times 10^{14} \mathrm{~g} \mathrm{~cm}^{-3}$ for model $\mathrm{s} 40 \mathrm{~A} 3 \mathrm{O} 09$ (leaving aside the exceptional models s40A2O13 and s40A2O15, which we will discuss separately later). This is another manifestation of the similarity of the two microphysical EoSs at subnuclear densities (see also Sec. IV C).
For slowly or at most moderately fast rotating models that undergo pressure-dominated bounce and whose center exceeds supernuclear density at (and also after) bounce, $\Delta \rho_{\text {max,b }}$ can amount up to $1.19 \times 10^{14} \mathrm{~g} \mathrm{~cm}^{-3}$ for model s11A1O01, the most slowly rotating model of the s11 model series. This strong impact of the EoS can be readily explained by the fact that at supernuclear densities the LS EoS is considerably softer than the Shen EoS. Figure 6 shows a difference in the adiabatic index $\gamma_{\text {eos }}$ between the two microphysical EoSs (for a representative model) of about $\Delta \gamma_{\text {eos }} \simeq-0.5$ at those densities, where nuclear forces have an essential impact on the EoS properties.

The large effect of the EoS seen in $\rho_{\text {max }, \mathrm{b}}$ in models where this quantity exceeds $\rho_{\text {nuc }}$ does not contradict our 
observation that the EoS has little impact on the collapse dynamics, since once the core plunges into the supernuclear density regime, where stronger differences in the two microphysical EoSs emerge, the mass $M_{\mathrm{ic}, \mathrm{b}}$ of the inner core at bounce is already fixed and the bounce dynamics (pressure dominated or centrifugal) is already determined.

The impact of the EoS on $\rho_{\max , \mathrm{b}}$ is also visualized in Fig. 8. As expected, for moderately or rapidly rotating models, whose central parts do not reach high supernuclear densities at bounce, the difference in $\rho_{\text {max,b }}$ gradually decreases. Figure 8 also reveals that the two models s40A2O13 and s40A2O15 (marked by two dark and light blue crosses at intermediate values of $\Omega_{\mathrm{c}, \mathrm{i}}$, respectively) are the ones that undergo a clear centrifugal bounce for both EoSs with the lowest value of $\Omega_{\mathrm{c}, \mathrm{i}}$ of all models with the $\mathrm{A} 2$ rotation profile.

The convergence of $\rho_{\text {max,b }}$ for the two microphysical EoS with increasing rotation can also be observed in the relative difference $\Delta \rho_{\max , \mathrm{b}, \mathrm{rel}}=\rho_{\text {max, } \mathrm{b}, \mathrm{LS}} / \rho_{\text {max, }, \text { Shen }}-1$ shown in Fig. 9, which starting from $\Delta \rho_{\text {max,b,rel }} \simeq$ $35-40 \%$ in the nonrotating limit first declines linearly with $\Omega_{\mathrm{c}, \mathrm{i}}$ until it levels off at roughly constant values. However, the largest values are obtained with $\Delta \rho_{\text {max,b,rel }} \simeq$ $40 \%$ and $48 \%$ for the rapidly rotating and centrifugally bouncing models s40A2O13 and s40A2O15, emphasizing their exceptional nature. This particular behavior results from a combination of two effects, exhibited by only these two models in our entire model set. First, when switching

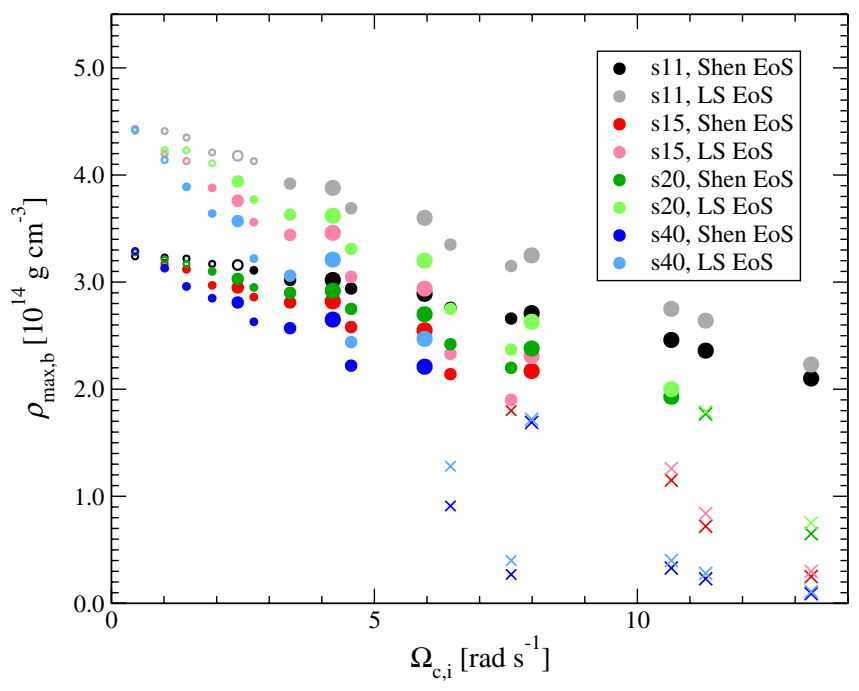

FIG. 8 (color online). Maximum density $\rho_{\text {max,b }}$ in the star at the time of bounce for all models versus the precollapse central angular velocity $\Omega_{\mathrm{c}, \mathrm{i}}$. For moderately fast or rapidly rotating models, which do not exceed nuclear density at bounce, $\rho_{\text {max,b }}$ is almost identical for the Shen EoS (dark hues) and the LS EoS (light hues), while for slowly rotating models the difference reaches $\Delta \rho_{\text {max }, \mathrm{b}} \simeq 10^{14} \mathrm{~g} \mathrm{~cm}^{-3}$ in the nonrotating limit. The progenitor mass, the EoS, the precollapse rotation parameter $A$, and the collapse dynamics are encoded as in Fig. 3. from the Shen EoS to the LS EoS the inner core mass $M_{\mathrm{ic}, \mathrm{b}}$ at bounce significantly decreases (see Fig. 4). Hence, in the LS EoS variant the two models experience weaker rotational support (in particular with the differential rotation parameter A2; see also Sec. IV C). Second, the two models bounce in a density regime (see Table III and Fig. 6), where the LS EoS exhibits a smaller $\gamma_{\text {eos }}$ than the Shen EoS, resulting in less pressure support when the LS EoS is used. The combination of weaker rotational support and pressure support when using the LS EoS can then readily explain the excess in $\rho_{\text {max,b,LS }}$ compared with $\rho_{\text {max,b,Shen }}$ in the two exceptional s40 models.

A higher value of the maximum density $\rho_{\max }$ in the collapsed core for the LS EoS is not limited to the time of bounce, but typically also remains in the nascent protoneutron star at later postbounce times, as shown in Fig. 10 for models representing the three collapse type and waveform subclasses (see Sec. IVA). Only very rapidly and thus centrifugally bouncing models such as model e20b in Fig. 10 have a time evolution of $\rho_{\max }$ that is practically independent of the chosen EoS.

We point out that in our discussion we always make use of the maximum density $\rho_{\max }$ instead of the central density $\rho_{\mathrm{c}}$, since, after bounce, some of the most rapidly rotating and thus centrifugally bouncing models develop a slightly toroidal density structure with an off-center density maximum that is at most $20 \%$ larger than $\rho_{\mathrm{c}}$. This is much less extreme than for models with the simplified hybrid EoS treatment, where the maximum density was found to be several orders of magnitude larger than the central density in extreme cases $[13,14]$.

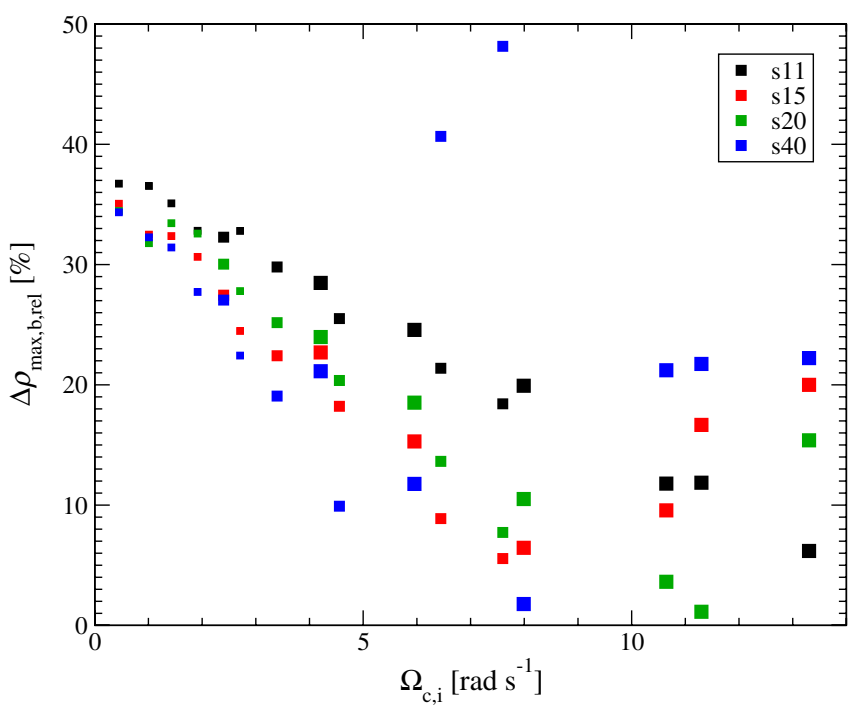

FIG. 9 (color online). Relative change $\Delta \rho_{\text {max,b,rel }}$ of the maximum density at bounce when changing from the Shen EoS to the LS EoS for all models versus the precollapse central angular velocity $\Omega_{\mathrm{c}, \mathrm{i}}$. The progenitor mass and the precollapse differential rotation parameter $A$ are encoded as in Fig. 3, while the collapse dynamics are not specified. 

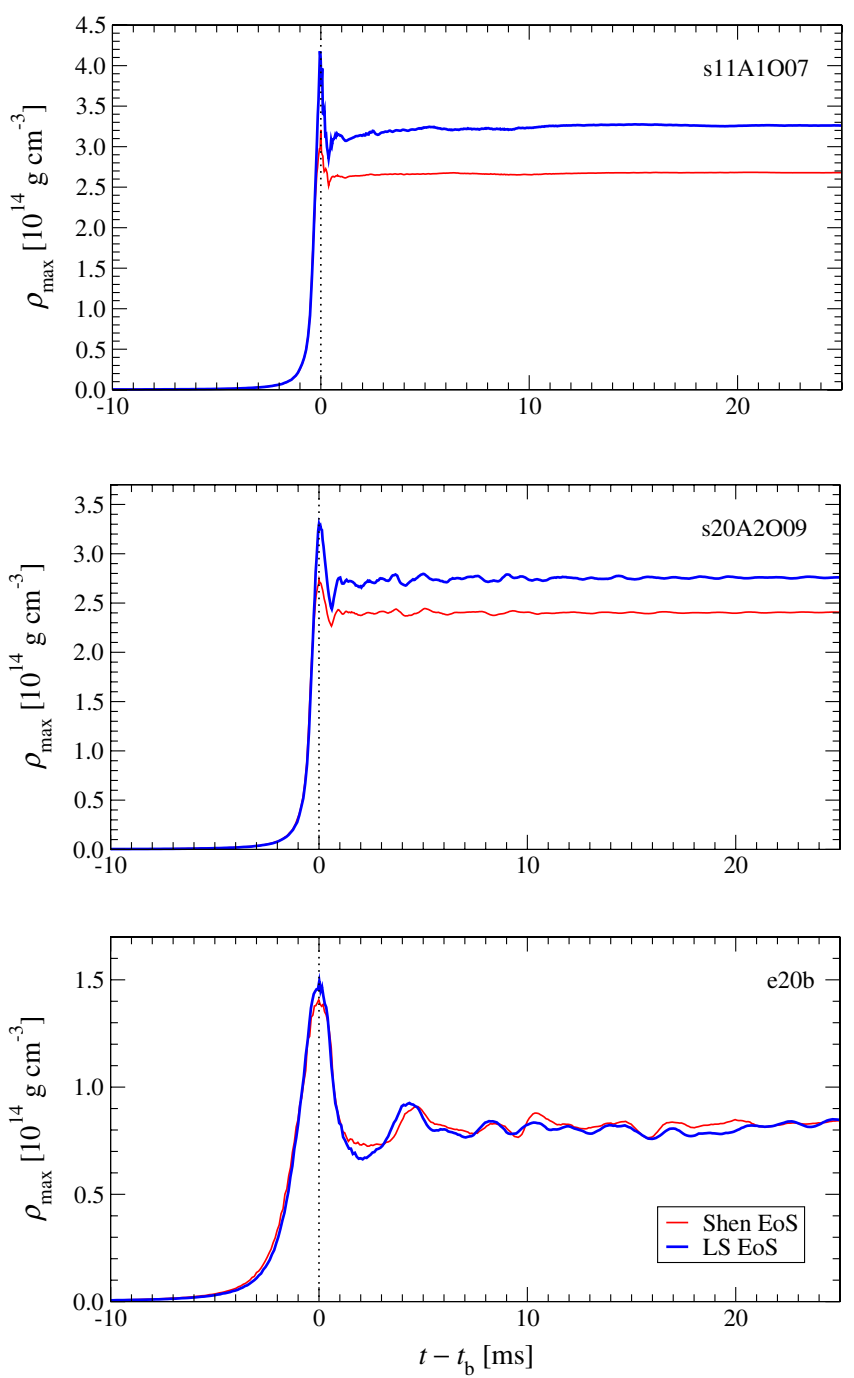

FIG. 10 (color online). Time evolution of the maximum density $\rho_{\max }$ for representative models with different precollapse rotation profiles using the Shen EoS (red lines) or LS EoS (blue lines). While models with at most moderate precollapse rotation rates (e.g., s11A1007 or s20A2O09) undergo a pressuredominated bounce at supernuclear densities, rapidly rotating models (e.g., e20b) experience a centrifugal bounce.

\section{B. Structure of the postcollapse core and peak waveform amplitude}

Since the LS EoS leads to higher central densities at bounce, one can on the one hand expect higher gravitational wave peak amplitudes in the burst signal from core bounce, as a denser and more compact core should yield in an increase of the contribution to the quadrupole moment from the central parts of the core. Furthermore, the associated shorter dynamical times also lead to a more rapid time variation in the quadrupole formula. On the other hand, the higher compactness of the inner core of a model run with the LS EoS results in lower densities at intermediate and large radii than in the less compact core of the corresponding model with the Shen EoS. In turn, this may lead to an effectively smaller total quadrupole moment and thus to a decrease in the signal amplitude compared with the counterpart model with the Shen EoS. We now test which of these two competing effects dominates in our models.

In Fig. 11, we show the peak value $|h|_{\max }$ of the gravitational wave amplitude for the burst signal from core bounce (see also Table III), where we neglect any possibly larger contributions at later times for models with strong prompt postbounce convection. For slowly or at most moderately rapidly rotating cores, $|h|_{\max }$ rises steeply with increasing $\Omega_{\mathrm{c}, \mathrm{i}}$ and covers a range of more than 2 orders of magnitude for our selection of initial models. For rapid rotation, when centrifugal forces become dynamically important and can be the dominant factor at bounce, the peak amplitude $|h|_{\max }$ saturates and even decreases again at very high $\Omega_{\mathrm{c}, \mathrm{i}}$. This behavior is a consequence of centrifugal support, which prevents such rapidly spinning cores from reaching high densities and more extreme compactness as well as being subject to short variations of the quadrupole moment (see also the discussion in Sec. VII and in [16]).

For each precollapse rotational configuration (i.e., at constant $\Omega_{\mathrm{c}, \mathrm{i}}$ and differential rotation parameter $A$ in Fig. 11), the value of $|h|_{\max }$ depends indirectly on the mass $M_{\text {prog }}$ of the progenitor via the mass $M_{\mathrm{ic}, \mathrm{b}}$ of the inner core at bounce. As already discussed in Sec. IV C, $M_{\mathrm{ic}, \mathrm{b}}$ does not depend in a monotonic way on $M_{\text {prog }}$, but for our standard model set increases in the order of the progenitor models s11, s20, s15, and s40. Therefore, for pressuredominated bounce models the amplitude of the gravitational wave signal directly scales with $M_{\mathrm{ic}, \mathrm{b}}$ in the obvious sense that more massive inner cores produce stronger gravitational wave emission.

What cannot be extracted from Fig. 11 is a clear effect of the choice of the EoS on $|h|_{\max }$, despite the strong difference in $\rho_{\text {max,b }}$ we observe between models using the Shen EoS and the LS EoS. When plotting the relative change $\Delta|h|_{\text {max,rel }}=|h|_{\text {max,LS }} /|h|_{\text {max,Shen }}-1$ obtained by changing from the Shen EoS to the LS EoS for the same initial model (as presented in Fig. 12), the majority of models shows a decrease of $|h|_{\max , \mathrm{LS}}$ compared with $|h|_{\max , \text { Shen }}$. Only six out of the 68 models (s11A2O05, s11A2O07, s11A3O05, s20A1O05, s20A1O07, and s40A3O09) listed in Table III exhibit a larger $|h|_{\max }$ when the LS EoS is used.

This behavior is similar to the situation discussed by Dimmelmeier et al. [14] who compared collapse dynamics and gravitational waveforms obtained from Newtonian and general relativistic collapse simulations with the simple hybrid EoS. They showed that for $|h|_{\max }$ the global density distribution in the core at bounce is decisive, not the local maximum density value. In their simulations, the general relativistic variants consistently produced an increase of $\rho_{\text {max,b }}$ compared with their Newtonian counterparts. Still, they found that the peak value $|h|_{\max }$ of the gravitational 


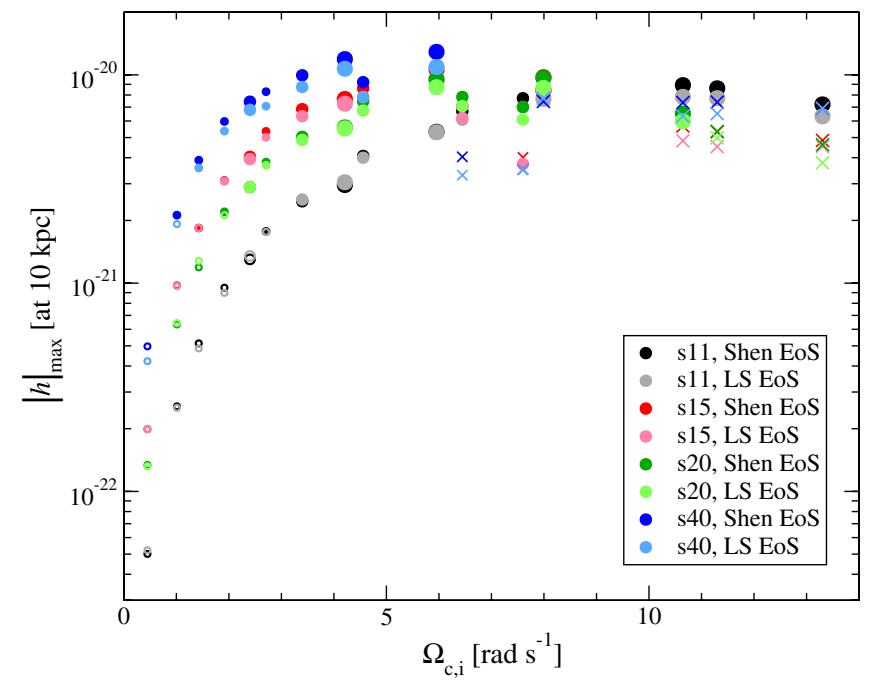

FIG. 11 (color online). Peak value $|h|_{\max }$ of the gravitational wave amplitude at $10 \mathrm{kpc}$ distance for the burst signal (neglecting possibly larger contributions from postbounce convection at later times) for all models versus initial precollapse central angular velocity $\Omega_{\mathrm{c}, \mathrm{i}}$. The progenitor mass, the EoS, the precollapse differential rotation parameter $A$, and the collapse dynamics are encoded as in Fig. 3.

wave amplitude actually decreases for most models when general relativistic effects are taken into account.

In [14], the negative $\Delta|h|_{\text {max, rel }}$ observed in many models when comparing Newtonian and relativistic simulations could be attributed to the "density crossing" that occurs at some radius inside the inner core at bounce: The general relativistic simulation of a model yields a higher density inside that (angle dependent, due to rotation) radius, while for larger distances from the center, $\rho$ is smaller compared with the Newtonian simulation. Here, we vary the EoS rather than the description of gravity, but we observe a very similar density crossing in models that show $|h|_{\text {max,LS }}<|h|_{\text {max,Shen }}$. In Fig. 13, we demonstrate this for models s20A3O09 (representative for a pressuredominated bounce) and s40A2O13 (representative for a centrifugal bounce).

Following the argument in [14], we plot the weighted density $\rho r^{2}$ in Fig. 14, since this is the relevant quantity in the integrand of the quadrupole gravitational wave formula. Although the larger $\rho r^{2}$ of the model with the LS EoS gives a larger quadrupole contribution out to the crossing radius, in most models the larger $\rho r^{2}$ in the outer parts of the core in the variant with the Shen EoS more than compensates this and ultimately leads to a larger integral quadrupole moment and, thus, to a stronger gravitational wave burst. We note that in [14], all models whose collapse type did not change exhibited lower peak waveform amplitudes $\left(\Delta|h|_{\text {max,rel }}<0\right)$ when going from Newtonian to general relativistic gravity. In contrast, going from the relatively stiff Shen EoS to the softer LS EoS results in a less clear trend with a few models exhibiting $\Delta|h|_{\text {max,rel }}>$

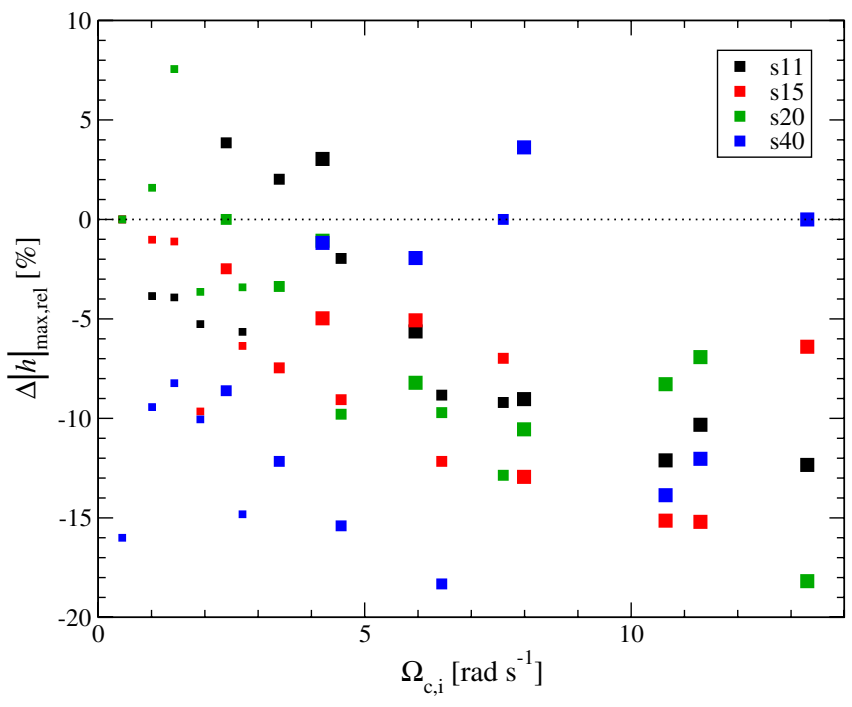

FIG. 12 (color online). Relative change $\Delta|h|_{\text {max,rel }}$ of the peak value $|h|_{\max }$ of the gravitational wave amplitude for the burst signal when changing from the Shen EoS to the LS EoS for all models versus the precollapse central angular velocity $\Omega_{\mathrm{c}, \mathrm{i}}$. The progenitor mass and the precollapse differential rotation parameter $A$ are encoded as in Fig. 3, while the collapse dynamics are not specified, as for some models it depends on the EoS.

0 . This suggests a less dramatic impact of a change from the Shen EoS to the LS EoS compared with altering the description of gravity.

For the small set of our models with $\Delta|h|_{\text {max,rel }}>0$ we are neither able to identify any obvious and systematic correlation with model parameters nor do we find any clear systematics of $\Delta|h|_{\text {max,rel }}>0$ with $\Delta \rho_{\text {max,b,rel }}$. It appears that the sign and magnitude of $\Delta|h|_{\text {max,rel }}$ depends sensitively and in a complicated way on the details of the collapse dynamics in each individual model. Hence, we can only explain why specific model differences in the density structure at bounce between the model variants with the Shen EoS and the LS EoS lead to an observed $\Delta|h|_{\text {max,rel }}$, but cannot predict $\Delta|h|_{\text {max,rel }}$ based on precollapse model parameters.

\section{Frequency spectrum of the waveform and variation with the equation of state}

In contrast to the somewhat ambiguous impact of the EoS on the peak waveform amplitude, the effect of replacing the Shen EoS with the LS EoS on the waveform peak frequency is unequivocal for models undergoing a pressure-dominated bounce. The increase in the maximum density at bounce in the models with the LS EoS always results in a shift of the main peak in the waveform spectrum to higher frequencies. In the center panel of Fig. 15, we plot the waveform spectrum (i.e., the Fourier transform $\hat{h}$ of $h$ ) for model s20A3009 as a representative pressuredominated bounce model. The spectrum of this model exhibits a distinct and narrow high-frequency peak at 


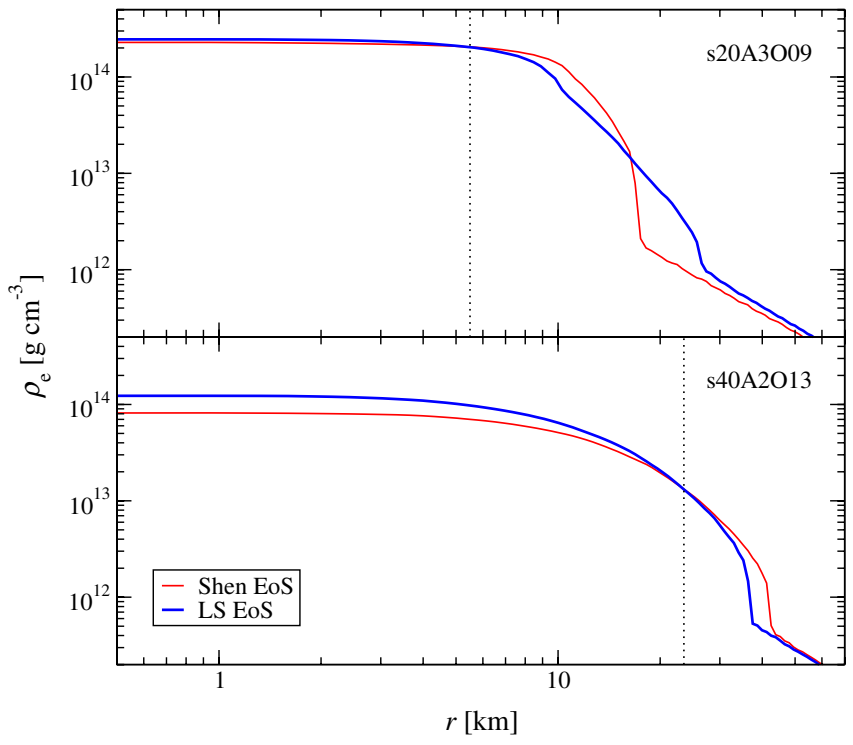

FIG. 13 (color online). Radial profiles of the density $\rho_{\mathrm{e}}$ in the equatorial plane at the time of bounce for model s20A3O09 (top panel) and model s40A2O13 (bottom panel) using the Shen EoS (red line) and LS EoS (blue line). In the central parts of the proto-neutron star (for these models at radii smaller than the crossing radius $r \simeq 5.5 \mathrm{~km}$ and $r \simeq 23.5 \mathrm{~km}$, respectively, marked by dotted lines) the LS EoS leads to higher densities.

$f_{\text {max,Shen }}=710 \mathrm{~Hz}$ when using the Shen EoS, while the calculation of the same model with the LS EoS results in a peak at $f_{\max , \mathrm{LS}}=744 \mathrm{~Hz}$. Thus, for this particular model, the change in EoS shifts the frequency associated with the bounce peak by $\Delta f_{\max }=+34 \mathrm{~Hz}$. We observe similar values for $\Delta f_{\max }$ in all models undergoing pressuredominated bounce.

At frequencies below about $200 \mathrm{~Hz}$, the waveform spectrum of s20A3O09 exhibits a plateau, which is due to the low-frequency contribution from prompt large-scale postbounce convection. Such a contribution is present in many models with slow to moderate rotation, but gradually decreases in magnitude and relevance with increasing rotation. As pointed out in Sec. IVA, our present numerical scheme has the tendency to overestimate prompt postbounce convection compared with full radiationhydrodynamics calculations.

The waveform of the slowly spinning model s20A1O05, whose spectrum is shown in the top panel of Fig. 15, is dominated by such prompt postbounce convective motions. Accordingly, for this model, there is a strong contribution to the spectrum at low frequencies, even exceeding the still clearly discernible bounce peak at high frequencies. Nevertheless, also in this case the shift of the high-frequency bounce peak when replacing the Shen EoS by the LS EoS is obvious and obeys the systematics discussed above.

With increasing rotation, centrifugal forces become more relevant and slow down the late phase of collapse and bounce. As a consequence, $f_{\max }$ always retreats to
PHYSICAL REVIEW D 78, 064056 (2008)

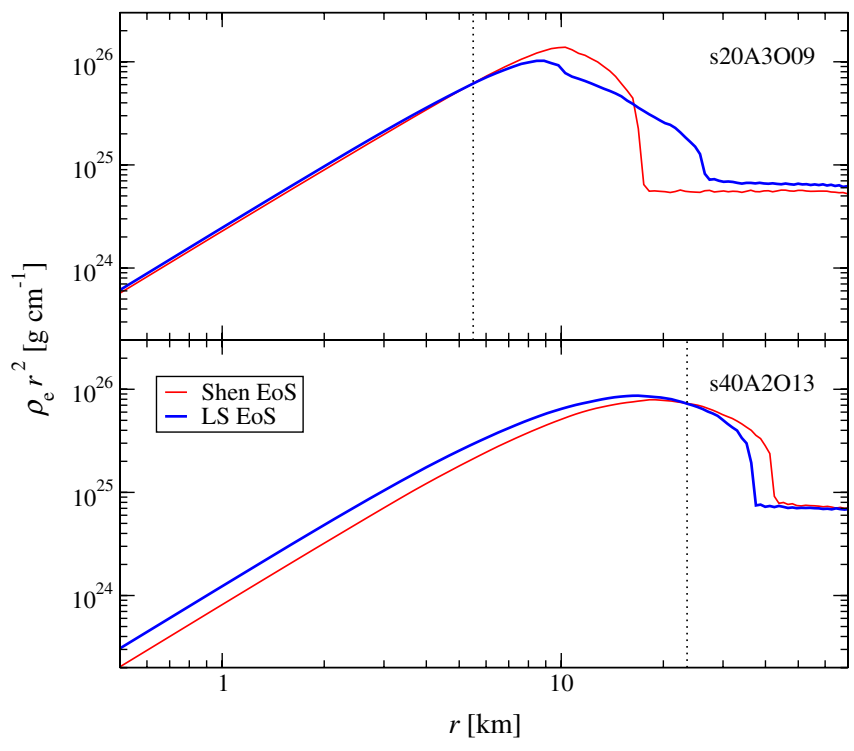

FIG. 14 (color online). Radial profiles of the weighted density $\rho_{\mathrm{e}} r^{2}$ in the equatorial plane at the time of bounce for model s20A3O09 (top panel) and model s40A2O13 (bottom panel) using the Shen EoS (red lines) and LS EoS (blue lines). The vertical lines mark the crossing radius.

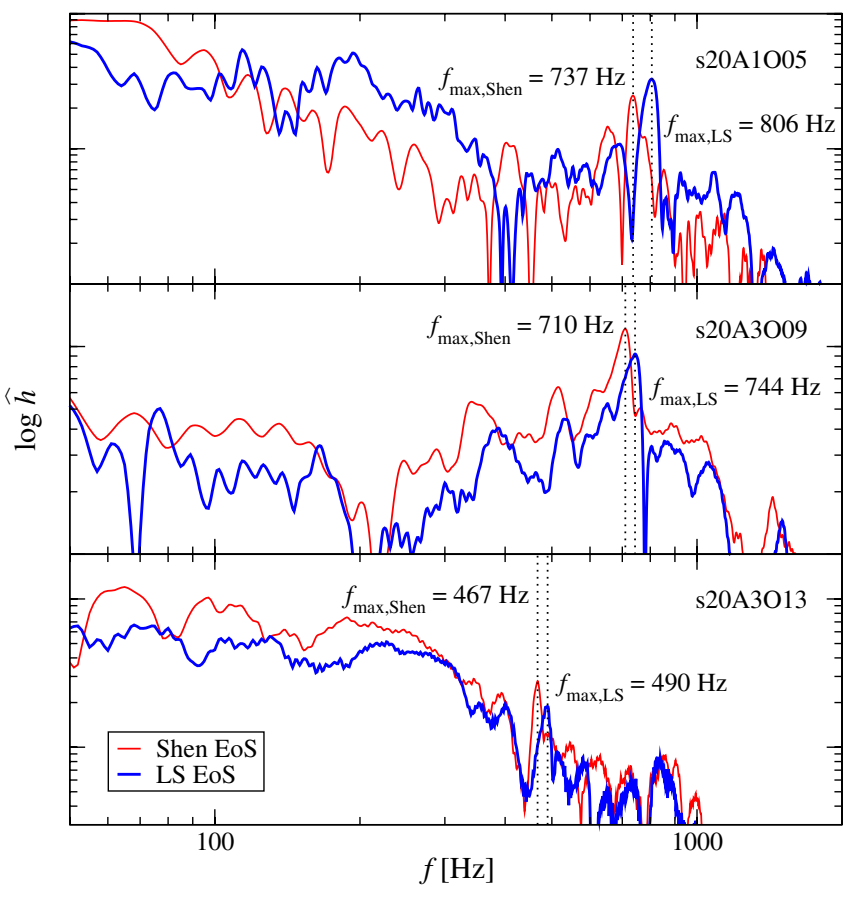

FIG. 15 (color online). Spectrum of the gravitational radiation waveform for model s20A1O05 (top panel), model s20A2O09 (center panel), and model s20A3O13 (bottom panel) using the Shen EoS (red line) and LS EoS (blue line). $\hat{h}$ is the Fourier transform in frequency space of the waveform amplitude $h$. The dotted lines mark the frequency $f_{\max }$ at the maximum of the waveform spectrum, neglecting low-frequency contributions. The scale of the vertical axis is 1 order of magnitude per major tick mark. 


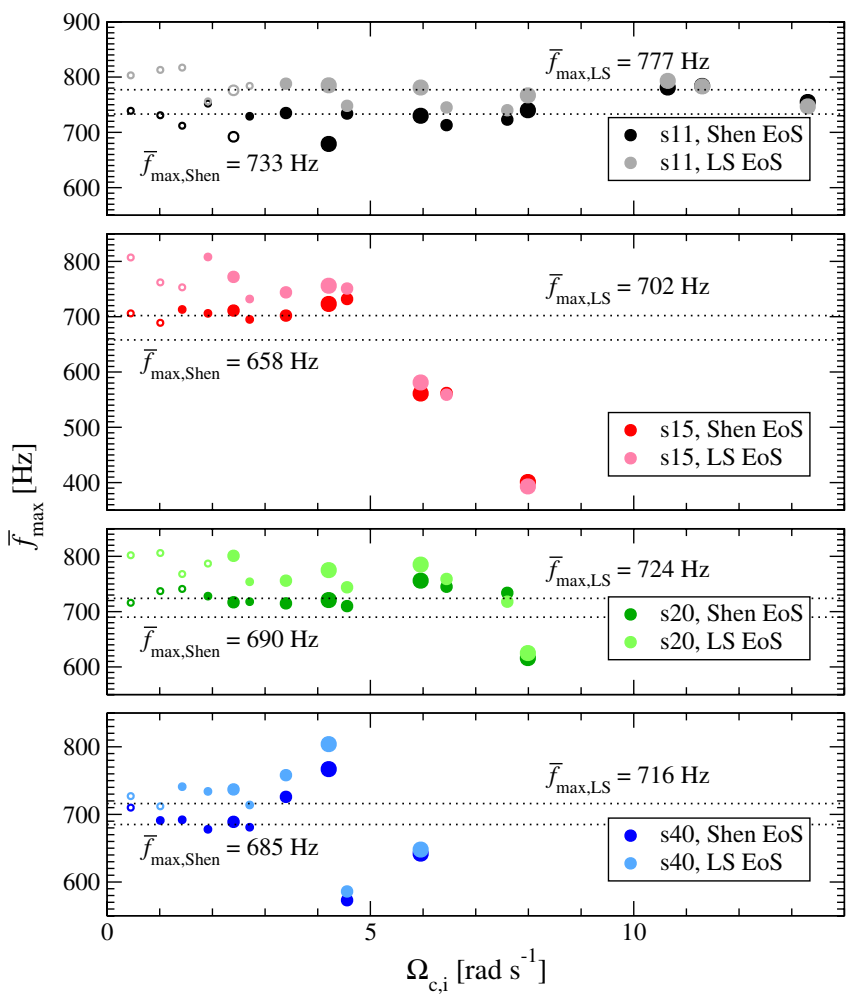

FIG. 16 (color online). Frequency $f_{\max }$ at the maximum of the waveform spectrum for all models with a given progenitor mass versus the precollapse central angular velocity $\Omega_{\mathrm{c}, \mathrm{i}}$. Only models that undergo pressure-dominated bounce are shown. The dotted lines mark the average $\bar{f}_{\max }$ when using the Shen EoS or LS EoS. The progenitor mass, the EoS, the precollapse differential rotation parameter $A$, and the collapse dynamics are encoded as in Fig. 3.

lower frequencies. This is apparent in the spectrum of the centrifugal bounce model s20A3O13 shown in the bottom panel of Fig. 15. For this model, one can still identify the high-frequency bounce peak, but now at significantly lower frequencies around $400-500 \mathrm{~Hz}$. Note that the lowfrequency quasicontinuous part of the spectrum in centrifugally bouncing models, such as s20A3O13, is due to rotationally slowed dynamics and stronger postbounce oscillations, and should not be confused with the lowfrequency contribution from prompt convection in slowly rotating models.

In Fig. 16, we plot $f_{\max }$ for all models that undergo pressure-dominated bounce and thus exhibit a clearly visible high-frequency peak in their spectra that can be associated with the gravitational wave burst from core bounce. For all models the systematic increase of $f_{\max }$ when changing from the Shen EoS to the LS EoS is apparent, and only for very few rapidly rotating models close to the threshold to centrifugal bounce the change of $f_{\max }$ becomes small. In Table IV, we summarize the arithmetic mean $\bar{f}_{\max }$ along with the respective absolute and relative differences between models using the Shen EoS and the LS EoS. Note
TABLE IV. Average $\bar{f}_{\max }$ of the frequency at the maximum of the waveform spectrum for all models with a given progenitor mass. $\Delta \bar{f}_{\text {max }}$ and $\Delta \bar{f}_{\text {max, rel }}$ are the absolute and relative change of the frequency average, respectively, when changing from the Shen EoS to the LS EoS.

\begin{tabular}{lcccc}
\hline \hline $\begin{array}{l}\text { Collapse } \\
\text { model set }\end{array}$ & $\begin{array}{c}\bar{f}_{\text {max,Shen }} \\
{[\mathrm{Hz}]}\end{array}$ & $\begin{array}{c}\bar{f}_{\text {max,LS }} \\
{[\mathrm{Hz}]}\end{array}$ & $\begin{array}{c}\Delta \bar{f}_{\max } \\
{[\mathrm{Hz}]}\end{array}$ & $\begin{array}{c}\Delta \bar{f}_{\text {max,rel }} \\
{[\%]}\end{array}$ \\
\hline s11 & 733 & 777 & 44 & 6.0 \\
s15 & 658 & 702 & 44 & 6.7 \\
s20 & 690 & 724 & 34 & 4.9 \\
s40 & 685 & 716 & 31 & 4.5 \\
\hline \hline
\end{tabular}

that when computing $f_{\max }$ we neglect the contribution below a cutoff frequency $f_{\text {cut }}=250 \mathrm{~Hz}$ in order to exclude any influence from the possibly unphysically strong and prolonged early postbounce convection.

In previous work [18], Dimmelmeier et al. discussed the detection prospects for the gravitational wave burst emitted in rotating core-collapse models based on the s20 progenitor and using the Shen EoS. To this end, they simulated a large set of models with varying precollapse rotation rates $\beta_{\mathrm{i}}$ in the range from $0.05-4 \%$, approximately logarithmically spaced in 18 steps for each of the three rotation profiles A1, A2, and A3. For the current work, we have repeated the calculations of this model set (which is extended in terms of precollapse rotation compared with our standard models stated in Table II, but limited to one progenitor), this time with the LS EoS. While the models with the Shen EoS that undergo pressure-dominated bounce have an arithmetic mean peak frequency $\bar{f}_{\text {max,Shen }} \sim 718 \mathrm{~Hz}[18]$, we find $\bar{f}_{\text {max,LS }} \sim 758 \mathrm{~Hz}$ when using the the LS EoS. Thus, for this particular model we set the average relative frequency shift amounts to $\Delta \bar{f}_{\text {max,rel }} \sim$ $5.6 \%$. Both the average peak frequencies and their change with EoS are consistent with what we find for our standard model set using the four different progenitors and a more restricted variety of precollapse rotation rates.

\section{DETECTION PROSPECTS FOR THE GRAVITATIONAL WAVE BURST SIGNAL}

In order to assess the detectability of the burst signal from core bounce, we compute the (detector-dependent) frequency-integrated characteristic signal frequency $f_{\mathrm{c}}$ and dimensionless characteristic gravitational wave amplitude $h_{\mathrm{c}}$ using Eqs. (22) and (23), respectively. We again exclude frequencies below $250 \mathrm{~Hz}$ from the integrals in an attempt to filter out dominant contributions from prompt postbounce convection in slowly rotating models. In Fig. 17, we plot $h_{\mathrm{c}}$ against $f_{\mathrm{c}}$ for the current LIGO detector [72] at a distance of $10 \mathrm{kpc}$. For comparison with the detector sensitivity, we include its rms strain sensitivity curve. Note that the total energy emitted in gravitational waves ranges from $E_{\mathrm{gw}} \sim 3.5 \times 10^{-10}$ to $5.3 \times 10^{-8}$ in units of 
$M_{\odot} c^{2}$ (including the contribution from convection) for our standard models.

The distribution of our standard set of models (as listed in Table II) in the $h_{\mathrm{c}}-f_{\mathrm{c}}$ plane of Fig. 17 obeys straightforward systematics. The clustering in frequency of the large number of models undergoing a pressure-dominated bounce (marked by circles in Fig. 17) is obvious. Very slowly rotating models, whose waveforms are dominated by the imprint of prompt postbounce convection (unfilled circles), exhibit the lowest values for $h_{\mathrm{c}}$, which increases with faster rotation (along arrow 1), reflecting that the inner core at bounce becomes more massive (cf. Secs. IV C and V B). Despite the frequency cut at $250 \mathrm{~Hz}$ in the integral for $f_{\mathrm{c}}$, the low-frequency contribution from convection in the spectrum leads to an $f_{\mathrm{c}}$ that is lower than the value obtained for more rapidly rotating models without significant postbounce convection (filled circles). For the latter model class, $h_{\mathrm{c}}$ simply grows with increasing precollapse rotation (along arrow 2), now at practically constant $f_{\mathrm{c}}$. Even for these models, $f_{\mathrm{c}}$ is always lower than the average peak frequency $\bar{f}_{\text {max }}$ of their waveform spectra, which amounts to $715 \mathrm{~Hz}$ for the 108 models of our standard model set (including the e15/e20 models) which exhibit a pressuredominated bounce. This is a consequence of the detector characteristics, whose maximum sensitivity is at much lower frequencies between 100 and $200 \mathrm{~Hz}$ and thus accordingly lowers $f_{\mathrm{c}}$ in comparison with a fiducial flat sensitivity curve.

For rapid rotation, the influence of centrifugal forces on the collapse dynamics manifests itself as a centrifugal

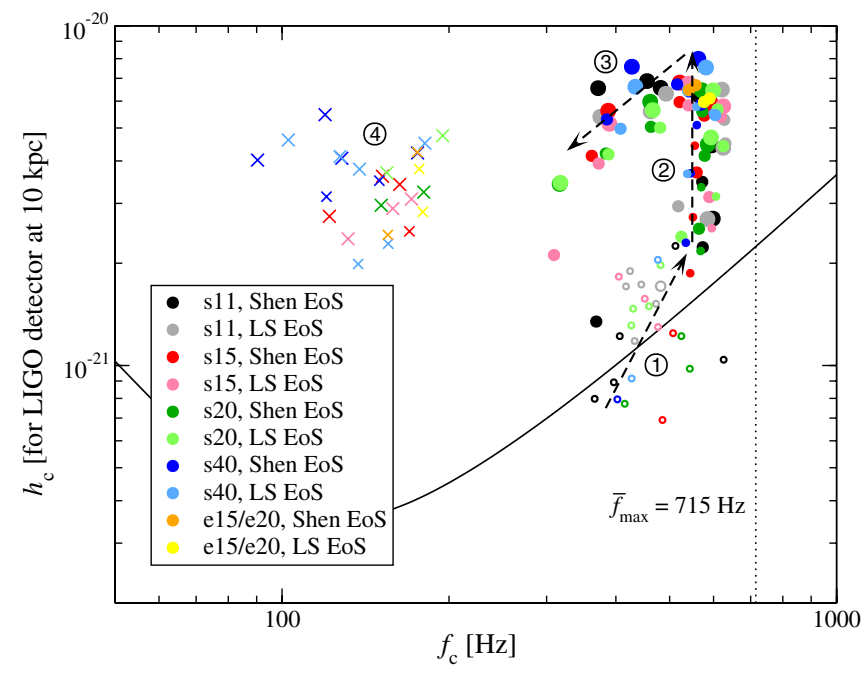

FIG. 17 (color online). Location of the gravitational wave burst signals from core bounce for all models (including the e15/e 20 models) in the $h_{\mathrm{c}}-f_{\mathrm{c}}$ plane relative to the sensitivity curves of the LIGO, assuming at a distance of $10 \mathrm{kpc}$. The meaning of the arrows 1,2 , and 3 as well as area 4 are explained in the main text. The dotted line marks the average $\bar{f}_{\text {max }}$ of the frequency at the maximum of the waveform spectrum. The progenitor model, the EoS, the initial rotation parameter $A$, and the collapse dynamics are encoded as in Fig. 3. barrier that limits the characteristic amplitude $h_{\mathrm{c}}$ (see also the discussion in Sec. VIIA and Fig. 11). Simultaneously, the characteristic frequency $f_{\mathrm{c}}$ moves to increasingly lower values as faster rotation slows down the collapse (along arrow 3). Models that rotate so rapidly that they undergo a purely centrifugal bounce (marked by cross symbols in Fig. 17) constitute a practically separate class (area 4 ) in the $h_{\mathrm{c}}-f_{\mathrm{c}}$ diagram somewhat below the maximum value of the amplitude $h_{\mathrm{c}}$, but at considerably lower frequencies $f_{\mathrm{c}}$.

For very rapidly rotating models the imprint of centrifugal effects on various waveform characteristics (such as $f_{\max }, f_{\mathrm{c}},|h|_{\max }$, or $h_{\mathrm{c}}$ ) is quite pronounced and permits one to infer on the precollapse rotational configuration in the case of a successful detection of gravitational waves from a core-collapse event. As already noted in [18], in the case of moderate or slow rotation, which is the astrophysically most probable case $[31,59]$, the insensitivity of the waveform's frequency characteristics to variations in the precollapse configuration significantly obstructs the "inversion problem" of gravitational wave detection, i.e., the constraining of physical parameters of the precollapse core or of the nascent proto-neutron star from a detected waveform, leaving only the (e.g., maximum or integrated characteristic) amplitude as an indicator of the rotational configuration. In addition, Fig. 16 also implies that it will

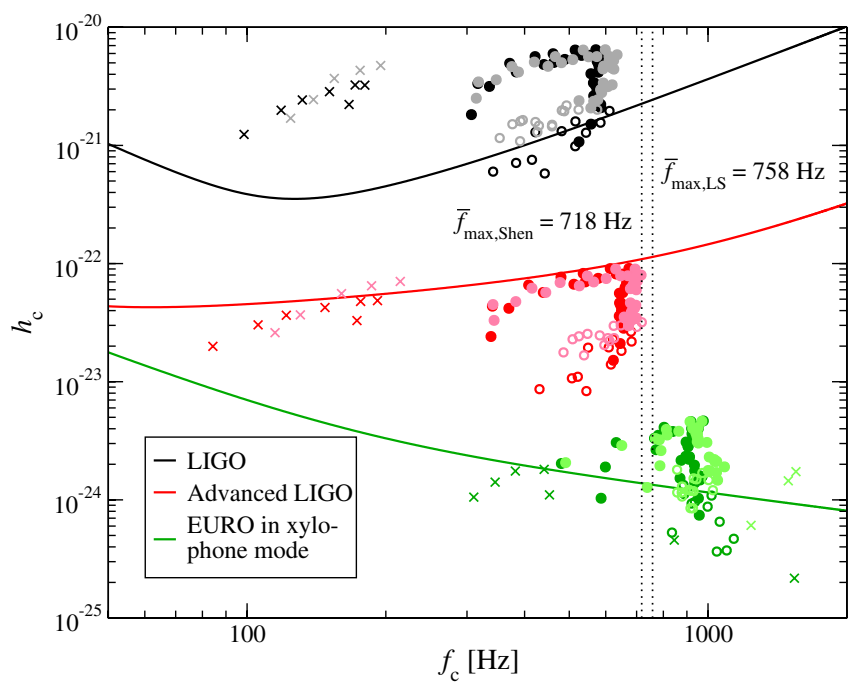

FIG. 18 (color online). Location of the gravitational wave burst signals from core bounce in the $h_{\mathrm{c}}-f_{\mathrm{c}}$ plane relative to the sensitivity curves of various interferometer detectors (as color-coded) for an extended set of models with the progenitor s20 using the Shen EoS (dark hues) or LS EoS (light hues). The sources are at a distance of $10 \mathrm{kpc}$ for LIGO, $0.8 \mathrm{Mpc}$ for Advanced LIGO, and $15 \mathrm{Mpc}$ for EURO. The dotted lines mark the average $\bar{f}_{\text {max }}$ of the frequency at the maximum of the waveform spectrum for the models when using the Shen EoS or LS EoS. Only the EoS and the collapse dynamics are encoded as in Fig. 3, but not the precollapse differential rotation parameter $A$. 
be very hard, if not impossible, to constrain other possibly unknown model parameters aside from rotation (such as EoS or progenitor mass) from the gravitational waveform of the burst signal from core bounce alone, since their effect on the burst waveform is small and no clear trends or systematics are discernible, which adds to the degeneracy of the inversion problem.

As an example, we again single out the impact of the EoS on the waveform frequency, while keeping the progenitor model s20 fixed. For the particular, extended set of models with many precollapse rotation rates already discussed in Sec. VC, we show in Fig. 18 the location of the waveform signals in the $h_{\mathrm{c}}-f_{\mathrm{c}}$ plane for initial LIGO at a distance of $10 \mathrm{kpc}$, Advanced LIGO in broadband tuning [72] at a distance of $0.8 \mathrm{Mpc}$, and the projected EURO detector in xylophone mode [73] at a distance of $15 \mathrm{Mpc}$ (cf. Fig. 4 in [18]). All 54 s20 models of [18] using the Shen EoS along with the newly computed corresponding models with the LS EoS are shown.

It is obvious that the spread within the group of models with either the Shen EoS or the LS EoS is larger than the variation due to a change in the EoS, since the effect of the EoS on the characteristic signal frequency $f_{\mathrm{c}}$ is small (comparable to $\Delta \bar{f}_{\text {max,rel }}$, corresponding to a change of a few percent). The two EoSs considered here bracket the range from rather soft (LS EoS) to rather stiff (Shen EoS), and therefore it is unlikely that employing a larger variety of nonzero-temperature nuclear EoSs would lead to any more optimistic conclusions.

Based on the relative positions of the models with respect to the individual detector sensitivities, from Fig. 18 we conclude (in agreement with previous work $[17,18])$ that initial-LIGO-class detectors are sensitive only to signals coming from an event in the Milky Way, while Advanced-LIGO-class observatories could marginally detect events from other galaxies in the Local Group (e.g., M31 Andromeda at $\sim 0.8 \mathrm{Mpc}$ distance). For the proposed EURO detector in xylophone mode, we expect a very high signal-to-noise ratio $\left(h_{\mathrm{c}}\right.$ divided by the detector sensitivity at $f_{\mathrm{c}}$ ). This detector could also observe many of the computed signals at a distance of $15 \mathrm{Mpc}$, i.e., in the Virgo cluster, for which one expects a favorably high event rate.

\section{ROTATION OF THE PROTO-NEUTRON STAR}

The calculations presented in this study impose axisymmetry, hence we are unable to track the development of rotationally induced nonaxisymmetric structures and dynamics. Nevertheless, we can utilize the results from our simulations to assess the possibility of rotational triaxial instabilities during the collapse and early postbounce phase. In this way we can (i) test the reliability of our present restriction to axisymmetry and (ii) put constraints on the relevance of the various types of such instabilities in a core-collapse event.
Nonaxisymmetric rotational instabilities in protoneutron stars have long been proposed as strong and potentially long-lasting sources of gravitational waves. In principle, the gravitational wave emission by a nonaxisymmetrically deformed proto-neutron star after bounce could easily exceed (see, e.g., [17,25]) in total emitted energy (and, hence, in characteristic strain $h_{\mathrm{c}}$ ) the gravitational wave burst from core bounce on which this paper is focussed.

In the context of classical Newtonian theory of fluid equilibria (see, e.g., [74]), MacLaurin spheroids (i.e., axisymmetric, rigidly rotating, equilibrium configurations of uniform density) become unstable to nonaxisymmetric deformation when a nonaxisymmetric configuration with lower total energy exists at a given rotation rate $\beta$. MacLaurin spheroids become dynamically unstable to deformation into Riemann ellipsoids at $\beta \gtrsim \beta_{\mathrm{dyn}}=27 \%$. At $\beta \gtrsim \beta_{\text {sec }}=14 \%$, they become secularly unstable to triaxial ellipsoidal deformation in the presence of dissipative processes (Jacobi ellipsoids via gravitational wave backreaction known as the Chandrasekhar-Friedman-Schutz (CFS) instability [75,76], or Dedekind ellipsoids via viscous processes). In both the dynamical and the secular case, the lowest-order deformation in terms of azimuthal nonaxisymmetric modes proportional to $\exp (\operatorname{im} \varphi)$ is the $m=2$ Kelvin (bar-) $f$-mode, where $\varphi$ is the azimuthal angle and the mode order $m$ is an integer.

Although Newtonian MacLaurin spheroids are highly idealized configurations, numerical studies (see, e.g., [77] and references therein) have shown that the above instability threshold $\beta_{\mathrm{dyn}}$ for the dynamical instability holds approximately even when differentially rotating compressible fluid configurations in general relativity are considered. The situation may be different for the gravitational radiation backreaction driven secular instability, since perturbative studies (see, e.g., [78]) predict an onset at significantly lower $\beta$ in general relativity than in the Newtonian case. However, fully relativistic nonlinear hydrodynamic studies of the secular instability remain yet to be carried out.

Recently, a new kind of dynamical rotational nonaxisymmetric instability at a value of $\beta$ much lower than the classical threshold has been discovered both in numerical and perturbative studies (see, e.g., $[17,22,25,79-85]$ and references therein). This low- $\beta$ instability (making the classical MacLaurin instability a "high"- $\beta$ instability) appears to amplify nonaxisymmetric modes at points where their pattern speed $\sigma_{m}$ (the eigenfrequency $\omega_{m}$ divided by the azimuthal mode order $m$ ) coincides with the local angular velocity of the fluid $[22,81-83]$.

\section{A. The rotational barrier in core collapse}

From first principles one can derive that the conservation of angular momentum during the collapse phase results in an increase of the angular velocity $\Omega$ of a representative 


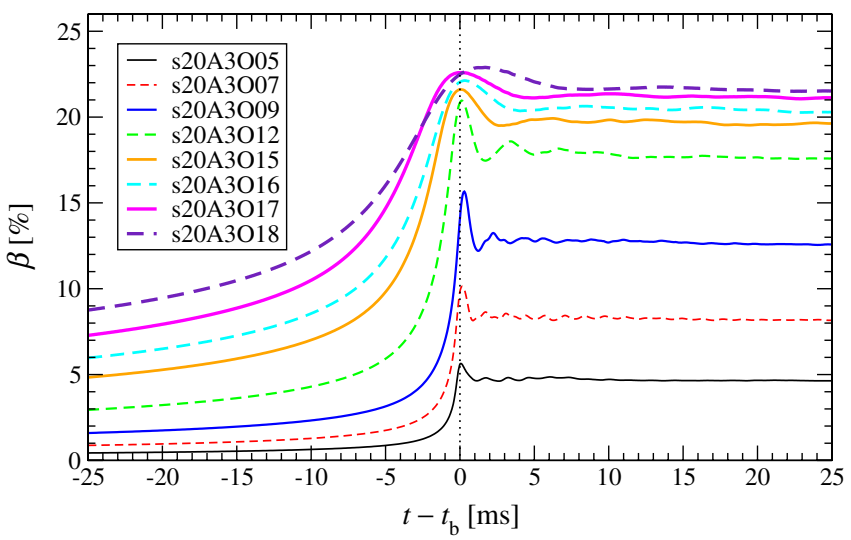

FIG. 19 (color online). Time evolution of the rotation rate $\beta$ around the time of core bounce for various models of the s20 progenitor series computed with the Shen EoS at fixed precollapse degree $A$ of differential rotation and varying the precollapse central angular velocity $\Omega_{\mathrm{c}, \mathrm{i}}$. Note that we have augmented this sequence by three extra models s20A3O16 to s20A3O18 (with $\beta_{\mathrm{i}}=3.00,3.50$, and 4.00 , respectively) not listed in Table III.

Lagrangian mass element proportional to $\varpi^{-2}$, where $\varpi=$ $r \sin \theta$ is the distance from the rotation axis. Setting for simplicity $\varpi$ equal to the spherical radial coordinate $r$ (which, of course, only holds in the equatorial plane), this translates into a scaling of the centrifugal force proportional to $r^{-3}$. The gravitational force, on the other hand,

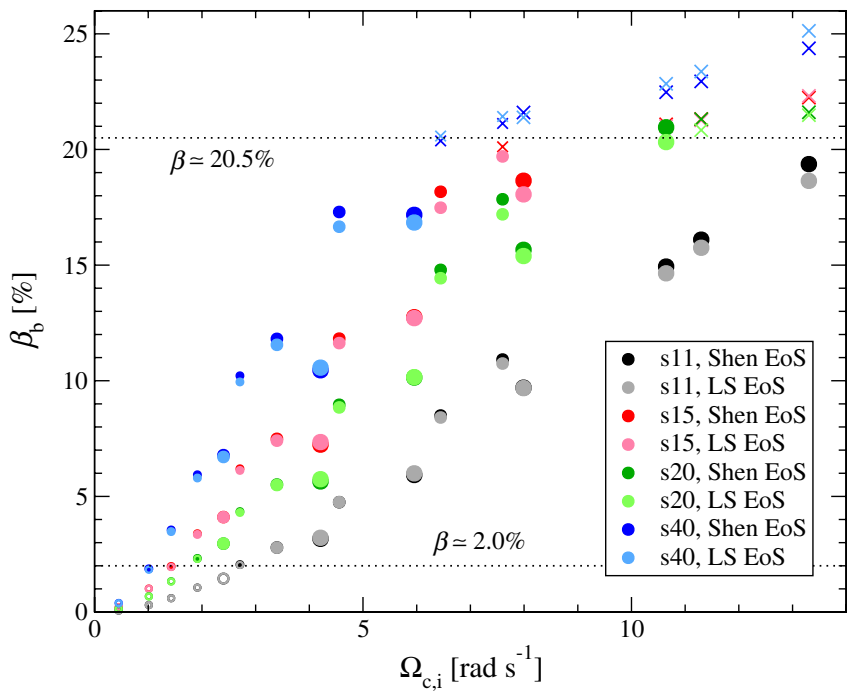

FIG. 20 (color online). Rotation rate $\beta_{\mathrm{b}}$ at the time of bounce for all models versus the precollapse central angular velocity $\Omega_{\mathrm{c}, \mathrm{i}}$. The progenitor mass, the EoS, the precollapse differential rotation parameter $A$, and the collapse dynamics are encoded as in Fig. 3. The lower horizontal line approximately separates pressure-dominated bounce models with and without strong prompt postbounce convection, while upper horizontal line marks the approximate transition between pressure-dominated bounce and centrifugal bounce. increases only like $r^{-2}$. Hence, even in this simple Keplerian picture, one may expect a dominance of the centrifugal force over gravity at sufficiently small $r$. In a more elaborate approach, employing sequences of Newtonian self-gravitating equilibrium spheroids, Tohline [86] demonstrated that such a rotational barrier at which the collapsing core becomes centrifugally stabilized indeed exists in the context of stellar core collapse. This rotational barrier marks the hard upper limit for the contraction of the inner core, hence also puts an upper limit $\beta_{\mathrm{rb}}$ on the rotation rate that can be reached when varying $\Omega_{\mathrm{c}, \mathrm{i}}$ for a given combination of precollapse degree of differential rotation and progenitor structure.

Tohline's qualitative conclusions have been confirmed by multiple numerical studies of rotating collapse (see, e.g., $[12-14,16,25]$ and our present work), while the quantitative results, in particular, the analytic critical rotation rate for centrifugal stabilization of collapse, do not hold for a dynamical collapse situation and must be determined via nonlinear hydrodynamic simulations [18].

In Fig. 19 we plot the time evolution of the rotation rate $\beta$ for a sequence of rotating collapse models with increasing precollapse central angular velocity $\Omega_{\mathrm{c}, \mathrm{i}}$ while all other model parameters are kept fixed. All models reach their maximum rotation rate $\beta_{\max }$ close to the time of core bounce, hence $\beta_{\max } \simeq \beta_{\mathrm{b}}$. After bounce, the inner core re-expands and settles into a new quasi-equilibrium configuration with $\beta_{\mathrm{pb}}<\beta_{\mathrm{b}}$. Slowly to moderately rapidly rotating models experience little rotational support, and in those cases $\beta_{\mathrm{b}}$ increases roughly linearly with $\Omega_{\mathrm{c}, \mathrm{i}}$

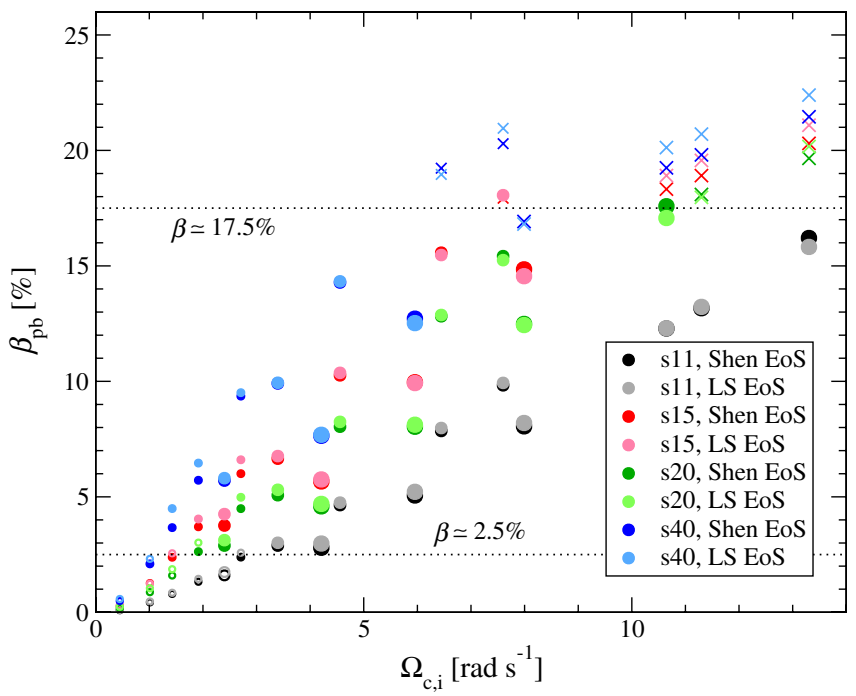

FIG. 21 (color online). Rotation rate $\beta_{\mathrm{pb}}$ in the late postbounce phase for all models versus the precollapse central angular velocity $\Omega_{\mathrm{c}, \mathrm{i}}$. The progenitor mass, the EoS, the precollapse differential rotation parameter $A$, and the collapse dynamics are encoded as in Fig. 3. As in Fig. 20, the horizontal lines again approximately mark the boundaries between different bounce dynamics. 
(see also Table III). For higher values of $\Omega_{\mathrm{c}, \mathrm{i}}$, centrifugal forces become relevant and $\beta_{\mathrm{b}}$ saturates at $\beta_{\mathrm{rb}}$ as the models start to bounce centrifugally. For the s20A3 sequence with the Shen EoS considered here we determine $\beta_{\mathrm{rb}}$ to be $\sim 23 \%$.

Figures 20 and 21 provide an overview of the dependences of $\beta_{\mathrm{b}}$ and $\beta_{\mathrm{pb}}$, respectively, on $\Omega_{\mathrm{c}, \mathrm{i}}$ for our entire model set as listed in Table III. Models that start out in essentially solid-body rotation (A1) never reach a $\beta_{\mathrm{b}}$ in excess of $\sim 10 \%$ (with the maximum obtained in model s40A1O13). With increasing $\Omega_{\mathrm{c}, \mathrm{i}}$ such rigidly rotating cores become eventually fully centrifugally supported already at the onset of collapse and do not collapse at all. Differentially rotating models may have higher values of $\Omega_{\mathrm{c}, \mathrm{i}}$ and thus a more rapidly rotating center, while the core is still allowed to collapse. As the collapse proceeds, electron capture reduces the pressure support and the size of the homologously collapsing inner core stays sufficiently small that centrifugal forces can become dynamically relevant only in the final phase of collapse (see the discussion in Sec. IV B). Thus, for our model set, the most differentially rotating configuration A3 leads to the highest values for $\beta_{\mathrm{b}}$ and $\beta_{\mathrm{pb}}$. A centrifugal bounce near the rotational barrier occurs only in a small subset of very rapidly $\left(\Omega_{\mathrm{c}, \mathrm{i}} \gtrsim 6.5 \mathrm{rad} \mathrm{s}^{-1}\right)$ and differentially (A2/A3) rotating models, generally at $\beta_{\mathrm{b}} \gtrsim 20.5 \%$.

At a fixed precollapse degree of differential rotation and $\Omega_{\mathrm{c}, \mathrm{i}}, \beta_{\mathrm{b}}$, and $\beta_{\mathrm{pb}}$ increase with a more massive and radially extended progenitor iron core (cf. Table I). This is analogous to the systematics found for the rotational enhancement of the inner core mass $M_{\mathrm{ic}, \mathrm{b}}$ at bounce (see Fig. 4).

The dependence of both $\beta_{\mathrm{b}}$ and $\beta_{\mathrm{pb}}$ on the EoS is small and shows little systematic trend. The Shen EoS, on the one hand, systematically yields a more massive and more extended inner core that bounces with more dynamically relevant angular momentum than one obtained with the LS EoS. The LS EoS, on the other hand, leads to more compact configurations, which provide for stronger centrifugal spinup in the final phase of collapse. The competition between these two effects results in the nonsystematic difference between the two EoSs seen in Figs. 20 and 21.

The centrifugal barrier is also evident in Fig. 22, where we plot the dependence of the peak value $|h|_{\max }$ of the gravitational wave burst against the rotation rate $\beta_{\mathrm{b}}$ at bounce. It is noteworthy that centrifugal effects are responsible for an upper limit in $|h|_{\max }$ even before the maximum rotation rate $\beta_{\mathrm{b}} \sim 25 \%$ is reached, which reflects the observation that the highest values of $|h|_{\max } \sim 10^{20}$ at $10 \mathrm{kpc}$ distance are obtained for models which still undergo a pressure-dominated bounce, albeit at rapid rotation with $\beta_{\mathrm{b}} \sim 10 \%$. Below these rotation rates, $|h|_{\max }$ scales linearly with $\beta_{\mathrm{b}}$ with remarkable precision, which is important information for the inversion problem in the case of a detection. We find a similar linear dependence of $|h|_{\max }$ on the postbounce rotation rate $\beta_{\mathrm{pb}}$. In that case, however,

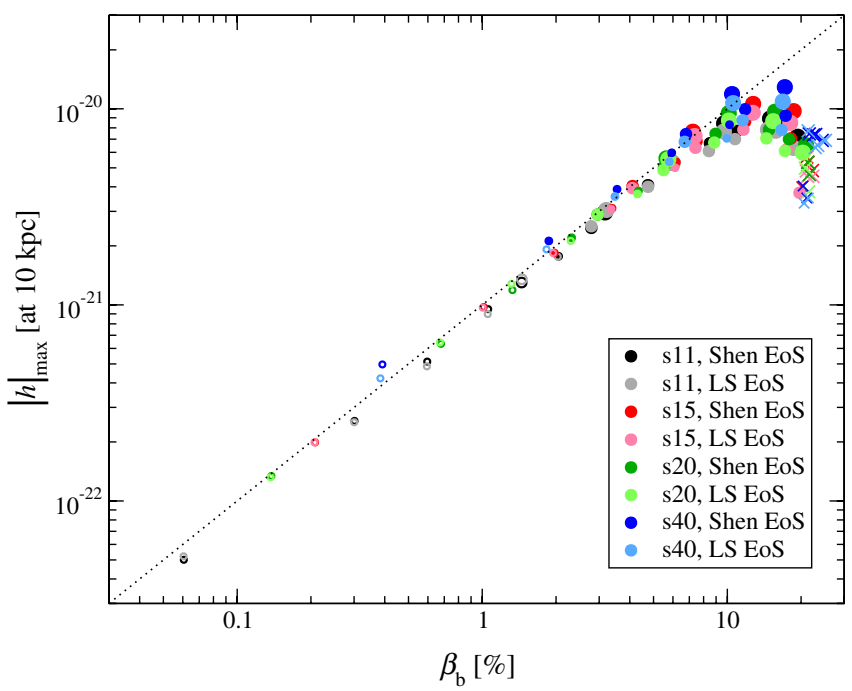

FIG. 22 (color online). Peak value $|h|_{\max }$ of the gravitational wave amplitude at $10 \mathrm{kpc}$ distance for the burst signal (neglecting possibly larger contributions from postbounce convection at later times) for all models versus the rotation rate $\beta_{\mathrm{b}}$ at the time of bounce. At slow to moderately rapid rotation, $|h|_{\max }$ is proportional to $\beta_{\mathrm{b}}$ to high accuracy (as marked by the dotted line with a slope of 1 in the log-log plot), while for $\beta_{\mathrm{b}} \gtrsim 10 \%$ centrifugal effects reduce $|h|_{\max }$.

the linear correlation is not as precise for low rotation rates (as $\beta_{\mathrm{pb}}$ is rather sensitive to angular momentum redistribution due to convection after core bounce) and, in addition, the scaling becomes approximately quadratic well before $|h|_{\max }$ reaches its upper limit.

\section{B. The prospects for dynamical high- $\beta$ instability in iron core collapse}

We find that none of our models surpass the threshold rotation rate $\beta_{\mathrm{dyn}}$ for the classical dynamical instability (see Table III). The overall largest $\beta$ of $\sim 25 \%$ is reached by model s40A3O15, which has the most massive and extended progenitor iron core (see Table I) in combination with the strongest precollapse degree of differential rotation and highest precollapse central angular velocity considered in this study. This value of $\beta_{\mathrm{b}} \sim 25 \%$ comes close to the numerically obtained instability threshold of $\beta_{\text {dyn }} \gtrsim$ $25.5 \%$ reported in [77], but is maintained only for a very short time, since the core rebounds and settles at a more expanded quasi-equilibrium state after bounce. Accordingly, its postbounce rotation rate $\beta_{\mathrm{pb}}$ is $\sim 22 \%$, and thus this model is unlikely to become subject to a dynamical high- $\beta$ bar-mode instability. As portrayed by Fig. 21, the models with less extreme precollapse conditions in general reach a $\beta_{\mathrm{pb}}$ significantly below $\sim 20 \%$.

Based on the results from our extensive set of simulations, we consider it unlikely that a proto-neutron star in nature develops a high- $\beta$ dynamical instability at or early after core bounce. On the other hand, during its cooling to 
the final cold and condensed neutron star, the proto-neutron star contracts, and, if angular momentum is conserved and not redistributed or shed by other means (see, e.g., the discussion in [31,59]), spins up on a timescale of seconds to minutes. While many of the proto-neutron stars in our model calculations could theoretically reach $\beta_{\mathrm{dyn}}$, it is, however, more likely that the secular instability driven by dissipation or gravitational radiation backreaction, which in proto-neutron stars has a growth timescale on the order of $1 \mathrm{~s}$ [87], will set in first, completely diminishing the chances for dynamical high- $\beta$ instability even in the most rapidly rotating proto-neutron stars.

Finally, we point out that it is in principle possible to construct precollapse conditions that lead to $\beta_{\mathrm{b}}$ and $\beta_{\mathrm{pb}}$ above $\beta_{\mathrm{dyn}}$. This may be achieved by increasing significantly the precollapse degree of differential rotation and $\Omega_{\mathrm{c}, \mathrm{i}}$ above the values used in our most extreme models. However, such configurations (including already the rotational setup A3 in our models) are very unlikely to arise in evolution scenarios of single massive stars, since stellar evolution proceeds sufficiently slowly for redistribution of angular momentum toward solid-body rotation to occur on nuclear-burning timescales $[31,43,88]$.

\section{Differential rotation in the proto-neutron star and its relevance for the low- $\beta$ dynamical instability}

The low- $\beta$ dynamical instability appears to develop exclusively in differentially rotating fluid bodies and has been reported to occur even for rotation rates as low as $\sim 1 \%$, provided the degree of differential rotation is sufficiently large [80].

The nature of the low- $\beta$ instability remains to be determined in detail, yet it has been suggested [81] that it is a type of dynamical shear instability that operates on the shear energy stored in differential rotation and radially redistributes angular momentum via the generation of an azimuthal (nonaxisymmetric, spiral) structure that propagates outward in radius $[25,83]$. In this picture, nonaxisymmetric structure is generated by transfer of rotational energy from the axisymmetric background fluid to an azimuthal fluid mode at the location where the background angular velocity matches the mode pattern speed (i.e., at the corotation point). This proposed corotation mechanism suggests a close relationship of the low- $\beta$ instability observed in simulations of stellar models with dynamical instabilities in disks such as those described by Papaloizou and Pringle [89].

The importance of differential rotation for the low- $\beta$ instability in stars can now be understood by the combination of two important factors: First, differential rotation provides the reservoir of shear energy that can be tapped to generate the nonaxisymmetric structure. Second, despite a relatively low global rotation rate $\beta$, differential rotation allows the central regions of a star to rotate sufficiently rapid to be in corotation with the lowest-order unstable modes that have pattern speeds of $\mathcal{O}\left(2 \pi / \tau_{\text {dyn }}\right)$, where

$$
\tau_{\mathrm{dyn}} \approx 2 \pi \sqrt{\frac{R^{3}}{G M}}
$$

is the dynamical timescale of the rotating star set by the Keplerian angular velocity [22,79].

Since solid-body rotation is the state of lowest rotational energy, neutron stars are very likely to become rigidly rotating within at most a few dissipative timescales during their post-supernova cooling evolution. Significant differential rotation may be expected in early merger remnants of binary neutron stars (e.g., [90]) and, importantly, is a consequence of rotating iron core collapse to a protoneutron star investigated in the present work.

In Fig. 23, we plot radial profiles of the angular velocity $\Omega$ in the equatorial plane at $20 \mathrm{~ms}$ after core bounce for several of our models. As a result of quasihomologous contraction, the near uniform precollapse rotational profile of the inner core is essentially frozen during collapse [59]. In the outer core, however, the collapse proceeds supersonically, resulting in differential rotation at equatorial radii $\gtrsim 10 \mathrm{~km}$. In all models shown in Fig. 23, $\Omega$ declines by about 2 orders of magnitude in the radial interval from 10 to $200 \mathrm{~km}$, and roughly obeys a power-law with an exponent in the range of -1.2 to -1.4 . Generally, a stronger degree of precollapse differential rotation leads to a steeper radial decline of $\Omega$ after bounce. When increasing $\Omega_{\mathrm{c}, \mathrm{i}}$ while keeping the degree of precollapse differential rotation fixed, the outer core regions experience more centrifugal support during collapse, resulting in a shallower postbounce slope for $\Omega$ (cf. model s20A2O15 in Fig. 23).

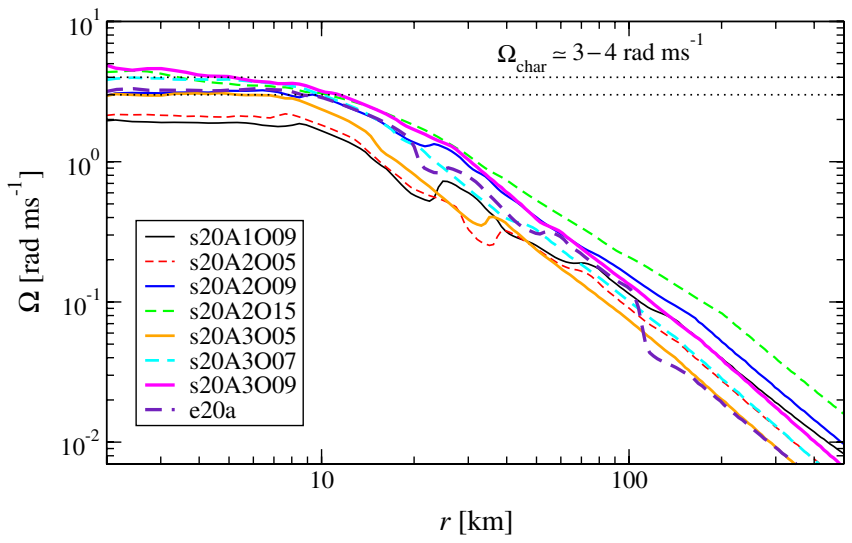

FIG. 23 (color online). Radial profile of the angular velocity $\Omega$ in the equatorial plane at $20 \mathrm{~ms}$ after the time of core bounce for a representative subset of the models listed in Table III. Note that the inner core is in approximate solid body rotation out to about $10 \mathrm{~km}$, while the outer parts of the proto-neutron star and the postshock region rotate strongly differentially. The dotted lines mark the approximate range for the characteristic angular frequency $\Omega_{\text {char }}$. 
In general, we find that the central angular velocity $\Omega_{\mathrm{c}}$ after bounce increases monotonically with the precollapse value $\Omega_{\mathrm{c}, \mathrm{i}}$. For our models we obtain values for $\Omega_{\mathrm{c}}$ in the nascent proto-neutron star between about 2 and $6 \mathrm{rad} \mathrm{ms}^{-1}$, which corresponds to central rotation periods of about 1 to $3 \mathrm{~ms}$. Assuming a mass range of the protoneutron star of $\sim 0.6$ to $0.8 M_{\odot}$ for the models considered here (see Fig. 4) and a fiducial radius of the inner core at bounce of $\sim 20 \mathrm{~km}$, we obtain dynamical times of $1.7-$ $2.0 \mathrm{~ms}$, which yield characteristic angular frequencies of $\Omega_{\text {char }} \approx 3-4 \mathrm{rad} \mathrm{ms}^{-1}$. Since the lowest-order unstable mode is likely to have a pattern speed of the order of $\Omega_{\text {char }}$, most models whose angular velocity we plot in Fig. 4 may indeed have corotation points with an unstable mode, hence could undergo a corotation-type low- $\beta$ instability. Slow rotators (with $\Omega_{\mathrm{c}, \mathrm{i}} \lesssim 2 \mathrm{rad} \mathrm{s}^{-1}$ ) do not appear to reach a sufficiently high angular velocity in the inner proto-neutron star core to have corotation points with potentially unstable modes in the first several tens of milliseconds after bounce. However, this may change at later times when the proto-neutron star contracts and spins up.

Finally, we point out that our discussion is based on a very rough estimate of the pattern speed for the lowestorder unstable azimuthal mode. More reliable estimates can be made via multidimensional perturbative analysis (see, e.g., [83] in the context of idealized models) or by performing a large set of numerical simulations in three dimensions, which we plan to carry out in a future study.

\section{SUMMARY AND CONCLUSIONS}

In this article we have presented results from a comprehensive set of collapse simulations of rotating stellar iron cores to proto-neutron stars, using the axisymmetric general relativistic hydrodynamics code COCONUT. Our simulations treat all the relevant physics of the collapse phase to good approximation. They include precollapse iron core profiles from stellar evolutionary calculations, a highly efficient approximate treatment of deleptonization, a microphysical finite-temperature $\mathrm{EoS}$, as well as neutrino pressure contributions. Magnetic fields are not included, since their relevance in the collapse and early postbounce phases is very likely negligible in cores with realistic precollapse fields $[5,31,33,37]$.

The focus of our study is on procuring accurate and reliable waveforms of the gravitational wave burst signal associated with core bounce and on understanding the dependence of the signal characteristics on progenitor star mass, precollapse rotational setup, and nuclear EoS. To this end, we have performed the to-date most extensive parameter study of this scenario, covering with more than 100 model calculations the parameter space spanned by (1) progenitor mass and model profile (zero-age main sequence masses from 11.2 to $40 M_{\odot}$, presupernova models with and without rotation), (2) rotational configuration (slow and uniform to rapid and differential rotation), and
(3) nuclear EoS prescription (from relatively soft to relatively stiff). Importantly, the parameter space encompasses and even goes beyond all precollapse rotational configurations that are deemed realistic in the context of collapsing massive stars.

A central result of this work is the finding that the gravitational wave burst from core bounce exhibits a generic waveform shape known as type I in the literature $[12,13]$, independent of the model parameters. The multiple centrifugal bounce dynamics and the corresponding type II waveform found in previous, technically less complete studies (see, e.g., $[12-14,16]$ ) do not occur in our models.

We have demonstrated that all models with precollapse core angular velocities $\Omega_{\mathrm{c}, \mathrm{i}}$ below $\sim 5 \mathrm{rad} \mathrm{s}^{-1}$ (corresponding periods longer than about $1 \mathrm{~s}$ ) reach nuclear densities and experience a core bounce predominantly due to nuclear pressure effects. More rapidly rotating cores develop sufficient rotational support during collapse to undergo either a mixture of centrifugal and pressure-dominated bounce or a single centrifugal bounce at subnuclear densities. Centrifugal hangup much below nuclear density or multiple, damped harmonic oscillatorlike centrifugal bounces do not occur. Therefore, these models also exhibit a type I waveform. The detailed analysis of the collapse dynamics presented in this paper reveals that the combined effects of general relativity and deleptonization lead to an increased destabilization of the collapsing core, result in a relatively small radius and mass $M_{\mathrm{ic}, \mathrm{b}}$ of the sonically connected inner core at bounce (but not small enough to show the type III waveform associated with rapid collapse found in some previous simplistic models), and diminish the dynamical importance of centrifugal forces during collapse.

The key parameter that determines the peak amplitude $|h|_{\max }$ of the gravitational wave burst has turned out to be the precollapse central angular velocity $\Omega_{\mathrm{c}, \mathrm{i}}$. Slowly rotating cores with $\Omega_{\mathrm{c}, \mathrm{i}} \leqslant 1 \mathrm{rad} \mathrm{s}^{-1}$ produce feeble peak amplitudes on the order of $10^{-22}$ at a distance of $10 \mathrm{kpc}$. More rapidly rotating cores with $1 \mathrm{rad} \mathrm{s}^{-1} \leqslant \Omega_{\mathrm{c}, \mathrm{i}} \leqslant 6 \mathrm{rad} \mathrm{s}^{-1}$ develop stronger quadrupole deformations and have a rotationally increased mass $M_{\mathrm{ic}, \mathrm{b}}$ at bounce, resulting in sizeable peak amplitudes in the range of $5 \times 10^{-22} \leqslant$ $|h|_{\max } \lesssim 10^{-20}$. The peaks of the waveform spectrum of such cores cluster in frequency space in the interval of $650-800 \mathrm{~Hz}$. At larger $\Omega_{\mathrm{c}, \mathrm{i}}$, centrifugal effects become strong, significantly decelerate collapse and bounce, and even lead to a purely centrifugal bounce in a subset of models. This results in a general decrease of $|h|_{\max }$ and a shift of the waveform's spectral peak to frequencies below $\sim 400 \mathrm{~Hz}$ at high $\Omega_{\mathrm{c}, \mathrm{i}}$.

We have also shown that, in addition to $\Omega_{\mathrm{c}, \mathrm{i}}$, the precollapse core mass in combination with the electron fraction sets the mass $M_{\mathrm{ic}, \mathrm{b}}$ of the inner core at bounce, is an important quantity influencing the strength of the gravita- 
tional burst. Since more massive progenitors generally (though with notable nonmonotonicity in the mass range from about 18 to $23 M_{\odot}$ ) form larger iron cores, we observe in our model series a general trend to bigger $M_{\mathrm{ic}, \mathrm{b}}$ and larger $|h|_{\max }$ with increasing progenitor mass if all other parameters are kept constant. For instance, the $40 M_{\odot}$ progenitor yields values of $|h|_{\max }$, which are up to 4 times larger than for the lower-entropy $11.2 M_{\odot}$ counterpart with the same rotational configuration.

The variations in the degree of differential rotation considered in this study have only a minor impact on the collapse dynamics and burst waveform amplitude. Increasing differential rotation at fixed $\Omega_{\mathrm{c}, \mathrm{i}}$ generally lowers the centrifugal support of outer core regions. However, since the dynamically most relevant inner core at bounce consists of only $\sim 0.5-1 M_{\odot}$ located within about $1000 \mathrm{~km}$ at the onset of collapse, the effects of differential rotation on the gravitational wave burst are small.

Our results further indicate that the nuclear EoS has little influence on the gravitational wave burst signal. For a given precollapse configuration, a softer nuclear EoS yields higher densities at bounce and postbounce times with shorter variation timescales of the quadrupole moment, but also leads to greater inner core compactness. In our simulations, the two effects generally cancel, leading to no systematic trend in the peak waveform amplitude $|h|_{\max }$ with the EoS. The peak of the waveform spectrum, however, shifts to higher frequencies in the case of a softer EoS. For the models considered here, this frequency shift amounts to $\sim 5.5 \%$ on average for models undergoing pressure-dominated bounce. It is significantly smaller for models bouncing at subnuclear densities under the influence of centrifugal effects.

If situated within our galaxy, a large fraction of our models are comfortably detectable by current gravitational wave detectors with a signal-to-noise ratio of up to 6 in the most optimistic case (which is obtained for the most rapidly rotating models that still undergo pressure-dominated core bounce). Advanced detectors could observe them easily out to $\sim 100 \mathrm{kpc}$ and up to several $10 \mathrm{Mpc}$ for third-generation detectors.

While such a gravitational wave signal may per se be detectable, the extraction of detailed physical information from the signal (i.e., solving the "inversion problem") from the signal will be a formidable task. The very generic morphology of the burst waveforms and the clustering in frequency space of most models make it seem unlikely that a pure waveform-template-based inversion (as, e.g., carried out in [91] using the waveforms of [16]) can be successful for determining key physical parameters to significant precision. Our results, however, suggest that based on $|h|_{\max }$ and the peak frequency $f_{\max }$ of the waveform spectrum alone, it should be possible to discriminate between purely pressure-dominated bounce (small to large $|h|_{\max }$ at frequencies $f_{\max }$ significantly above $500 \mathrm{~Hz}$ ) and centrifu- gal bounce (large $|h|_{\max }$ at frequencies $f_{\max }$ significantly below $500 \mathrm{~Hz}$ ). Furthermore, we find that for not too rapid rotation $|h|_{\max }$ can be directly used to extract the rotation rate $\beta_{\mathrm{b}}$ at bounce to good precision.

Making use of the extensive set of postbounce rotational configurations obtained with our simulations, we have also studied the prospects for the development of nonaxisymmetric rotational instabilities in nascent proto-neutron stars. We find that the rotational barrier imposed by centrifugal forces prohibits the spinup to rotation rates necessary for the classical dynamical bar-mode instability at high values of $\beta$. We find, however, that a large subset of our postbounce models exhibits sufficiently differential and rapid rotation to become subject to the recently discovered low- $\beta$ instability. Still, three-dimensional simulations as in $[17,19,22,38]$ will be necessary to provide conclusive tests of our predictions. Furthermore, the interaction and competition of the low- $\beta$ instability and other instabilities operating on the shear energy of differential rotation, for instance the magneto-rotational instability (see, e.g., [33,92]), remain to be studied.

Finally, we point out that this study may be regarded as part - with the presently highest level of sophisticationof a multidecade effort of our groups [11-14,16-18] to provide reliable estimates for the gravitational wave burst emission associated with rotating core collapse and core bounce. The waveforms presented here are for the first time not only accurate (i.e., numerically converged), but reliable and robust, since our calculations take into account all the necessary physics, including general relativity, deleptonization, and a microphysical EoS. All waveforms are available for download in various formats in a publicly accessible waveform catalog [42].

We point out that the gravitational wave emission process considered in this work operates at measurable strength only if the progenitor core is rotating a lot more rapidly than expected for ordinary iron cores (see, e.g., $[31,59])$. In slowly rotating core-collapse supernovae, turbulent convective overturn, instabilities of the accretion shock, and, possibly, proto-neutron star pulsations are likely to be the dominant emission processes of gravitational waves. The characteristics of these emission processes are not as well understood and will require more extensive and precise modeling to provide accurate estimates of the complete gravitational wave signature of corecollapse supernovae.

\section{ACKNOWLEDGMENTS}

It is a pleasure to thank Shizuka Akiyama, David Arnett, Adam Burrows, Luc Dessart, Pablo Cerdá-Durán, Ian Hawke, Alex Heger, Ewald Müller, Shangli Ou, José Pons, Erik Schnetter, Ed Seidel, Bernard Schutz, Todd Thompson, Joel Tohline, and Burkhard Zink for helpful comments and inspiring discussions. This work was supported by the Deutsche Forschungsgemeinschaft through 
the Transregional Collaborative Research Centers Contract No. SFB/TR 27 "Neutrinos and Beyond," Contract No. SFB/TR 7 "Gravitational Wave Astronomy," and the Cluster of Excellence EXC 153 "Origin and Structure of the Universe" [93], by the DAAD and IKY (IKYDA German-Greek research travel grant), and by the European Network of Theoretical Astroparticle Physics Contract No. ENTApP ILIAS/N6 under Contract No. RII3-CT-2004-506222. H.D is supported by a Marie
Curie Intra-European Fellowship within the 6th European Community Framework Programme under Contract No. IEF 040464, and C.D. O. by the Joint Institute for Nuclear Astrophysics (JINA) under NSF Sub-Award No. 61-5292UA of NFS Award No. 86-6004791. The authors wish to thank the Max Planck Institute for Gravitational Physics and the John von Neumann-Institut für Computing (NIC) in Jülich where the calculations presented in this paper were performed.
[1] P. Goldreich and S. V. Weber, Astrophys. J. 238, 991 (1980).

[2] A. Yahil, Astrophys. J. 265, 1047 (1983).

[3] H.-T. Janka, K. Langanke, A. Marek, G. Martínez-Pinedo, and B. Müller, Phys. Rep. 442, 38 (2007).

[4] A. Burrows, L. Dessart, C. D. Ott, and E. Livne, Phys. Rep. 442, 23 (2007).

[5] A. Burrows, L. Dessart, E. Livne, C. D. Ott, and J. Murphy, Astrophys. J. 664, 416 (2007).

[6] S. W. Bruenn, C. J. Dirk, A. Mezzacappa, J. C. Hayes, J. M. Blondin, W. R. Hix, and O. E. B. Messer, J. Phys. Conf. Ser. 46, 393 (2006).

[7] M. Liebendörfer, O. E. B. Messer, A. Mezzacappa, S. W. Bruenn, C. Y. Cardall, and F.-K. Thielemann, Astrophys. J. Suppl. Ser. 150, 263 (2004).

[8] S.E. Woosley and J.S. Bloom, Annu. Rev. Astron. Astrophys. 44, 507 (2006).

[9] L. Dessart, A. Burrows, E. Livne, and C.D. Ott, Astrophys. J. Lett. 673, L43 (2008).

[10] P. Aufmuth and K. Danzmann, New J. Phys. 7, 202 (2005).

[11] E. Müller, New J. Phys. 114, 53 (1982).

[12] R. Mönchmeyer, G. Schäfer, E. Müller, and R. E. Kates, Astron. Astrophys. 246, 417 (1991).

[13] T. Zwerger and E. Müller, Astron. Astrophys. 320, 209 (1997).

[14] H. Dimmelmeier, J. Font, and E. Müller, Astron. Astrophys. 393, 523 (2002).

[15] K. Kotake, S. Yamada, and K. Sato, Phys. Rev. D 68, 044023 (2003).

[16] C.D. Ott, A. Burrows, E. Livne, and R. Walder, Astrophys. J. 600, 834 (2004).

[17] C. D. Ott, H. Dimmelmeier, A. Marek, H.-T. Janka, I. Hawke, B. Zink, and E. Schnetter, Phys. Rev. Lett. 98, 261101 (2007).

[18] H. Dimmelmeier, C. D. Ott, H.-T. Janka, A. Marek, and E. Müller, Phys. Rev. Lett. 98, 251101 (2007).

[19] M. Rampp, E. Müller, and M. Ruffert, Astron. Astrophys. 332, 969 (1998).

[20] E. Müller, M. Rampp, R. Buras, H.-T. Janka, and D. H. Shoemaker, Astrophys. J. 603, 221 (2004).

[21] M. Shibata and Y.-I. Sekiguchi, Phys. Rev. D 71, 024014 (2005).

[22] C. D. Ott, S. Ou, J. E. Tohline, and A. Burrows, Astrophys. J. Lett. 625, L119 (2005).
[23] C. D. Ott, A. Burrows, L. Dessart, and E. Livne, Phys. Rev. Lett. 96, 201102 (2006).

[24] L. Blanchet, Living Rev. Relativity 9, 4 (2006), www.livingreviews.org/lrr-2006-4.

[25] C. D. Ott, Ph.D thesis, Universität Potsdam, Germany, 2006, opus.kobv.de/ubp/volltexte/2007/1298.

[26] W. Hillebrandt and R.G. Wolff, in Nucleosynthesis: Challenges and New Developments, edited by W.D. Arnett and J. W. Truran (University of Chicago Press, Chicago, 1985), p. 131.

[27] K. A. van Riper and J. M. Lattimer, Astrophys. J. 249, 270 (1981).

[28] H. Komatsu, Y. Eriguchi, and I. Hachisu, Mon. Not. R. Astron. Soc. 237, 355 (1989).

[29] H.-T. Janka, T. Zwerger, and R. Mönchmeyer, Astron. Astrophys. 268, 360 (1993).

[30] M. Obergaulinger, M. A. Aloy, H. Dimmelmeier, and E. Müller, Astron. Astrophys. 457, 209 (2006).

[31] A. Heger, S.E. Woosley, and H. C. Spruit, Astrophys. J. 626, 350 (2005).

[32] K. Kotake, S. Yamada, K. Sato, K. Sumiyoshi, H. Ono, and H. Suzuki, Phys. Rev. D 69, 124004 (2004).

[33] P. Cerdá-Durán, J. A. Font, and H. Dimmelmeier, Astron. Astrophys. 474, 169 (2007).

[34] T. Nakamura and H. Sato, Phys. Lett. A 86, 318 (1981).

[35] M. Shibata and Y.-I. Sekiguchi, Phys. Rev. D 69, 084024 (2004).

[36] P. Cerdá-Durán, G. Faye, H. Dimmelmeier, J. A. Font, J. M. Ibáñez, E. Müller, and G. Schäfer, Astron. Astrophys. 439, 1033 (2005).

[37] M. Obergaulinger, M. A. Aloy, and E. Müller, Astron. Astrophys. 450, 1107 (2006).

[38] C.D. Ott, H. Dimmelmeier, A. Marek, H.-T. Janka, B. Zink, I. Hawke, and E. Schnetter, Classical Quantum Gravity 24, S139 (2007).

[39] H. Shen, H. Toki, K. Oyamatsu, and K. Sumiyoshi, Prog. Theor. Phys. 100, 1013 (1998).

[40] M. Liebendörfer, Astrophys. J. 633, 1042 (2005).

[41] H. Dimmelmeier, C. D. Ott, H.-T. Janka, A. Marek, and E. Müller, in Gravitational Waves and Experimental Gravity, edited by J. Dumarchez and J. Trân Than Vân (Thê Giói Publishers, Vietnam, 2007), p. 59.

[42] www.mpa-garching.mpg.de/rel_hydro/wave_catalog. shtml. 
[43] A. Heger, N. Langer, and S. E. Woosley Astrophys. J. 528, 368 (2000).

[44] J. M. Lattimer, and F. D. Swesty, Nucl. Phys. A535, 331 (1991).

[45] J. W. York in Sources of Gravitational Radiation, edited by L. L. Smarr (Cambridge University Press, Cambridge, 1979), p. 83.

[46] F. Banyuls, J. A. Font, J. M. Ibáñez, J. M. Martí, and J. A. Miralles, Astrophys. J. 476, 221 (1997).

[47] J. A. Isenberg, Int. J. Mod. Phys. D 17, 265 (2008).

[48] J. R. Wilson, G. J. Mathews, and P. Marronetti, Phys. Rev. D 54, 1317 (1996).

[49] J. W. York, Phys. Rev. Lett. 26, 1656 (1971).

[50] A. Garat and R. H. Price, Phys. Rev. D 61, 124011 (2000).

[51] G. B. Cook, S. L. Shapiro, and S. A. Teukolsky, Phys. Rev. D 53, 5533 (1996).

[52] H. Dimmelmeier, N. Stergioulas, and J. A. Font, Mon. Not. R. Astron. Soc. 368, 1609 (2006).

[53] H. Shen, H. Toki, K. Oyamatsu, and K. Sumiyoshi, Nucl. Phys. A 637, 435 (1998).

[54] J. M. Lattimer, C. J. Pethick, D. G. Ravenhall, and D. Q. Lamb, Nucl. Phys. A 432, 646 (1985).

[55] A. Marek, H.-T. Janka, R. Buras, M. Liebendörfer, and M. Rampp, Astron. Astrophys. 443, 201 (2005).

[56] G. Martínez-Pinedo, M. Liebendörfer, and D. Frekers, Nucl. Phys. A777, 395 (2006).

[57] K. Langanke and G. Martínez-Pinedo, Nucl. Phys. A673, 481 (2000).

[58] S.E. Woosley, A. Heger, and T. A. Weaver, Rev. Mod. Phys. 74, 1015 (2002).

[59] C. D. Ott, A. Burrows, T. A. Thompson, E. Livne, and R. Walder, Astrophys. J. Suppl. Ser. 164, 130 (2006).

[60] S.E. Woosley and A. Heger, Astrophys. J. 637, 914 (2006).

[61] M. Shibata and Y.-I. Sekiguchi, Phys. Rev. D 68, 104020 (2003).

[62] H. Dimmelmeier, J. Novak, J. A. Font, J. M. Ibáñez, and E. Müller, Phys. Rev. D 71, 064023 (2005).

[63] K. S. Thorne, Rev. Mod. Phys. 52, 299 (1980).

[64] A. Nagar, O. Zanotti, J. A. Font, and L. Rezzolla, Phys. Rev. D 75, 044016 (2007).

[65] K. S. Thorne, in 300 Years of Gravitation, edited by S. W. Hawking and W. Israel (Cambridge University Press, Cambridge, 1987), p. 330.

[66] J. A. Font, Living Rev. Relativity 6, 4 (2003), www.livingreviews.org/lrr-2003-4.

[67] J. M. Hyman, Technical Report COO-3077-139, ERDA Mathematics and Computing Laboratory, Courant
Institute of Mathematical Sciences (New York University, New York, 1976).

[68] C. L. Fryer and A. Heger, Astrophys. J. 541, 1033 (2000).

[69] R. Buras, M. Rampp, H.-T. Janka, and K. Kifonidis, Astron. Astrophys. 447, 1049 (2006).

[70] L. Dessart, A. Burrows, C. D. Ott, E. Livne, S.-C. Yoon, and N. Langer, Astrophys. J. 644, 1063 (2006).

[71] A. Burrows and J. M. Lattimer, Astrophys. J. 270, 735 (1983).

[72] D. Shoemaker (private communication).

[73] www.astro.cardiff.ac.uk/geo/euro.

[74] S. Chandrasekhar, Ellipsoidal Figures of Equilibrium (Yale University Press, New Haven, 1969).

[75] S. Chandrasekhar, Astrophys. J. 161, 561 (1970).

[76] J. L. Friedman and B.F. Schutz, Astrophys. J. 222, 281 (1978).

[77] L. Baiotti, R. De Pietri, G. M. Manca, and L. Rezzolla, Phys. Rev. D 75, 044023 (2007).

[78] S. M. Morsink, N. Stergioulas, and S.R. Blattnig, Astrophys. J. 510, 854 (1999).

[79] J. M. Centrella, K. C. B. New, L. L. Lowe, and J. D. Brown, Astrophys. J. 550, L193 (2001).

[80] M. Shibata, S. Karino, and Y. Eriguchi, Mon. Not. R. Astron. Soc. 343, 619 (2003).

[81] A. L. Watts, N. Andersson, and D. I. Jones, Astrophys. J. Lett. 618, L37 (2005).

[82] S. Ou and J. E. Tohline, Astrophys. J. 651, 1068 (2006).

[83] M. Saijo and S. Yoshida, Mon. Not. R. Astron. Soc. 368, 1429 (2006).

[84] B. Zink, N. Stergioulas, I. Hawke, C. D. Ott, E. Schnetter, and E. Müller, Phys. Rev. D 76, 024019 (2007).

[85] P. Cerdá-Durán, V. Quilis, and J. A. Font, Comput. Phys. Commun. 177, 288 (2007).

[86] J. E. Tohline, Astrophys. J. 285, 721 (1984).

[87] D. Lai, in Astrophysical Sources for Ground-Based Gravitational Wave Detectors, edited by J. M. Centrella (AIP Press, New York, 2001), p. 246.

[88] R. Hirschi, G. Meynet, and A. Maeder, Astron. Astrophys. 425, 649 (2004).

[89] J. C. B. Papaloizou and J. E. Pringle, Mon. Not. R. Astron. Soc. 213, 799 (1985).

[90] S. Rosswog and M. B. Davies, Mon. Not. R. Astron. Soc. 334, 481 (2002).

[91] T. Summerscales, A. Burrows, C. D. Ott, and L. S. Finn, Astrophys. J. 678, 1142 (2008).

[92] S. A. Balbus and J.F. Hawley, Astrophys. J. 376, 214 (1991).

[93] www.universe-cluster.de. 